\title{
Crosstalk between autophagy inhibitors and endosome-related secretory pathways: a challenge for autophagy-based treatment of solid cancers
}

\author{
Martina Raudenska ${ }^{1,2,3 \dagger}$, Jan Balvan ${ }^{1,2,3 \dagger}$ and Michal Masarik ${ }^{1,2,3,4,5^{*}}$ (D)
}

\begin{abstract}
Autophagy is best known for its role in organelle and protein turnover, cell quality control, and metabolism. The autophagic machinery has, however, also adapted to enable protein trafficking and unconventional secretory pathways so that organelles (such as autophagosomes and multivesicular bodies) delivering cargo to lysosomes for degradation can change their mission from fusion with lysosomes to fusion with the plasma membrane, followed by secretion of the cargo from the cell. Some factors with key signalling functions do not enter the conventional secretory pathway but can be secreted in an autophagy-mediated manner.

Positive clinical results of some autophagy inhibitors are encouraging. Nevertheless, it is becoming clear that autophagy inhibition, even within the same cancer type, can affect cancer progression differently. Even nextgeneration inhibitors of autophagy can have significant non-specific effects, such as impacts on endosome-related secretory pathways and secretion of extracellular vesicles (EVs). Many studies suggest that cancer cells release higher amounts of EVs compared to non-malignant cells, which makes the effect of autophagy inhibitors on EVs secretion highly important and attractive for anticancer therapy. In this review article, we discuss how different inhibitors of autophagy may influence the secretion of EVs and summarize the non-specific effects of autophagy inhibitors with a focus on endosome-related secretory pathways. Modulation of autophagy significantly impacts not only the quantity of EVs but also their content, which can have a deep impact on the resulting pro-tumourigenic or anticancer effect of autophagy inhibitors used in the antineoplastic treatment of solid cancers.
\end{abstract}

Keywords: Autophagy, Autophagy inhibitors, Cancer, Endosomes, Multivesicular bodies, Extracellular vesicles, Exosomes, Amphisomes, Non-conventional secretory pathways

\section{Introduction}

Autophagy is a highly evolutionarily conserved mechanism best known for its role in organelle and protein turnover, cell quality control, and metabolism.

\footnotetext{
*Correspondence: masarik@med.muni.cz

${ }^{\dagger}$ Martina Raudenska and Jan Balvan contributed equally to this work.

${ }^{3}$ Department of Pathological Physiology, Faculty of Medicine, Masaryk University, Kamenice 5, CZ-625 00 Brno, Czech Republic

Full list of author information is available at the end of the article
}

Lysosome-mediated degradative autophagy provides a source of nutrients and energy by digestion of cytoplasmic elements and serves for the clearance of toxic protein aggregates and defective organelles [1]. This recycling pathway can also profoundly affect cellular specialization and differentiation [2], protein trafficking, and unconventional secretion $[3,4]$. Three types of autophagy have been observed in mammalian cells: macroautophagy, microautophagy, and chaperone-mediated autophagy. During microautophagy the lysosomal membrane original author(s) and the source, provide a link to the Creative Commons licence, and indicate if changes were made. The images or other third party material in this article are included in the article's Creative Commons licence, unless indicated otherwise in a credit line to the material. If material is not included in the article's Creative Commons licence and your intended use is not permitted by statutory regulation or exceeds the permitted use, you will need to obtain permission directly from the copyright holder. To view a copy of this licence, visit http://creativecommons.org/licenses/by/4.0/. The Creative Commons Public Domain Dedication waiver (http://creativeco mmons.org/publicdomain/zero/1.0/) applies to the data made available in this article, unless otherwise stated in a credit line to the data. 
insulates the autophagic cargo directly, whereas, during macroautophagy, double-membrane structures called autophagosomes are formed to deliver autophagic cargo to endosomes or lysosomes. Macroautophagy also participates in the specific degradation of organelles during mitophagy, ribophagy, or pexophagy. Chaperone-mediated autophagy involves the selective degradation of KFERQ-like motif-bearing proteins supplied to the lysosomes via chaperone HSC70 and other cochaperones (e.g. CHIP, HOP, and heat shock protein 40). Internalization of cargo into lysosomes is managed via the receptor lysosome-associated membrane protein type 2A (LAMP2A) [5]. In this review article, we will focus on macroautophagy (hereafter referred to as autophagy).

Endocytosis is the process by which cells can sequester substances from the external environment by engulfing them in vesicles. Endocytosis includes the clathrin-dependent pathway as well as clathrin-independent pathways such as phagocytosis, pinocytosis, raft-mediated endocytosis, and ARF6-dependent internalization. As well as autophagy, endocytosis can culminate into lysosomal degradation, but here the cargo is internalized from the plasma membrane, not from the cytoplasm [6]. After internalization, the cargo is sorted by highly dynamic compartments, called early endosomes (EEs), marked by unique adaptor proteins, effector proteins, and small Rab GTPases such as RAB4, RAB5, early endosomal antigen-1 (EEA1), VPS34, and SNAREs. EEs are the major cellular sorting platform as they can mature into endosomes destined for various cellular fates. EE cargo can be recycled to the plasma membrane via recycling endosomes, transported to or from the Golgi apparatus via the retromer complex, or routed to lysosomes via multivesicular bodies (MVBs)/late endosomes [7]; see Fig. 1. Autophagy and endocytic pathways cooperate at some stages and share many components of the molecular machinery.

Recent studies also show that there are many interconnections between autophagy, exosome/amphisome biogenesis, and exocytosis of extracellular vesicles (EVs) $[3,4]$. To release exosomes and/or amphisomes, several steps need to be performed such as the biogenesis of intraluminal vesicles (ILVs) in MVBs, transport of MVBs to autophagosomes or the plasma membrane and fusion of MVBs and/or amphisomes with the plasma membrane [8]. These steps are deeply affected by molecules of autophagy machinery $[9,10]$ including many Rab GTPases such as RAB7, RAB11, RAB35, RAB27A, RAB27B and the vesicle-associated membrane protein 7 (VAMP7) [11]. RAB7 and RAB11 also participate in autophagosome formation and RAB7 has a key role in autophagosome maturation (for further details see the chapter Autophagy and MVBs) [12]. Consequently, autophagy can have both stimulatory or inhibitory effects on the secretion of extracellular vesicles (EVs) and these effects will probably be deeply context-dependent. This can partially explain the double-edged sword character of autophagy in cancer progression. In this review article, we discuss how different inhibitors of autophagy may influence the secretion of EVs and summarize the nonspecific effects of autophagy inhibitors with a focus on endosome-related secretory pathways. Modulation of autophagy significantly impacts not only the quantity of EVs but also their content which can have a deep impact on the resulting pro-tumourigenic or anticancer effect of autophagy inhibitors used in antineoplastic treatment of solid cancers.

\section{Basic molecular mechanism of degradative macroautophagy}

Macroautophagy (hereafter referred to as autophagy) is a process in which double-membrane vesicles (autophagosomes) are formed around a segment of the cytoplasm. Once autophagosomes are formed, they can either fuse with lysosomes and form autolysosomes, or they can bring together organelles of endosomal origin to form amphisomes with a single limiting membrane [13-15].

Autophagosome formation goes through five main stages - initiation, nucleation, elongation, fusion, and cargo degradation. The detailed molecular mechanism of autophagy has been extensively reviewed in Klionsky et al. [16], therefore, we present here only the basic molecular mechanisms important for the understanding of the effects of autophagy inhibitors on macroautophagy.

The initiation phase of autophagy is preceded by the inhibition of mTORC1. mTORC1 is inhibited by cellular and environmental stresses that are incompatible with continued growth, such as glucose or amino acid deprivation, DNA damage, or hypoxia. mTORC1 consists of three core components: mTOR (highly conserved serine/ threonine-protein kinase belonging to the PI3K-related kinase family), RAPTOR (regulatory protein associated with mTOR responsible for mTORC1 localization and substrate recruitment), and mLST8. In addition to these three core components, mTORC1 also contains two inhibitory subunits DEPTOR (DEP domain-containing mTOR interacting protein) and PRAS40 (proline-rich Akt substrate of $40 \mathrm{kDa}$ ) [17]. A decrease in cellular energy activates the stress-responsive metabolic regulator AMPK (AMP-activated protein kinase), which inhibits mTORC1 indirectly through activation of Tuberous Sclerosis Complex (TSC), or directly through the phosphorylation of RAPTOR by protein kinase A (PKA) $[18,19]$. TSC suppresses mTORC1 by converting Rheb GTPase from an active GTP-bound form to an inactive GDP-bound state. The TSC complex requires G3BPs (Ras 


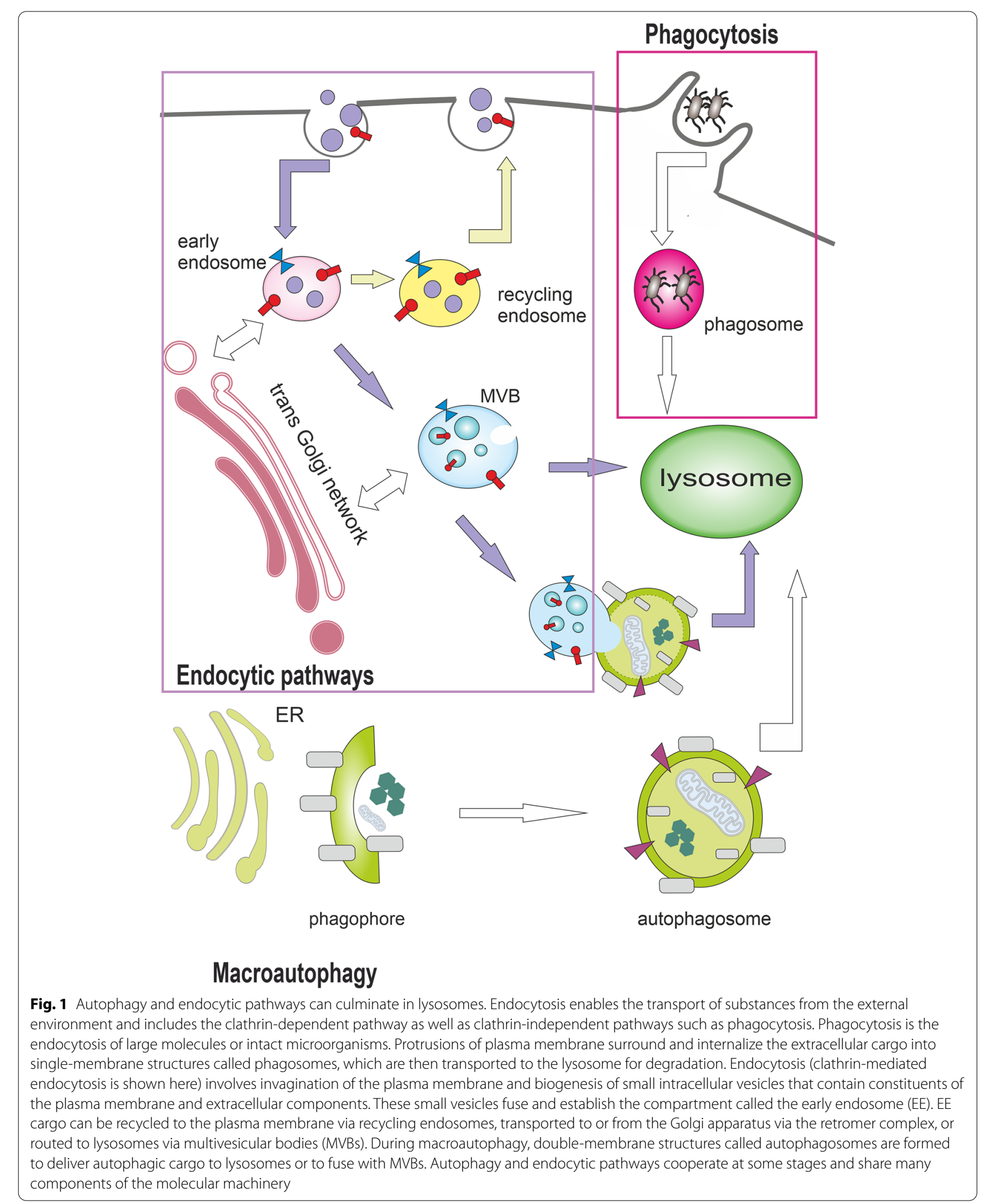


GTPase-activating protein-binding proteins) as its lysosomal tether [20].

For sensing the levels of nutrients, the presence of mTORC1 on lysosomes is crucial [21]. In response to amino acids, mTORC1 present on lysosomes can be activated by Rag and Rheb guanosine triphosphatases (GTPases) and can trigger anabolic processes [22]. A key player in Rag-mTORC1 activation is the vacuolar $\mathrm{H}^{+}$ ATPase (V-ATPase) that couples ATP hydrolysis (peripheral V1 domain) to proton translocation through the lysosomal membrane (integral V0 domain) to acidify the lysosomes and enable their degradative functions. When the level of amino acids in the lumen of lysosomes is low, the V-ATPase turns off the activity of Rag GTPases. In contrast, when amino acids are abundant, the V-ATPase undergoes conformational changes leading to the activation of Rag heterodimers and the recruitment of mTORC1 to lysosomes [23] (see Fig. 2). Lysosomes are usually localized closer to the plasma membrane when amino acids and growth factors are abundant. On the contrary, when they are limited, the Rap1-GTPases imprison lysosomes in the perinuclear region and reduce lysosome abundance, therefore reducing the lysosomal surface available for mTORC1 activation, which suppresses mTORC1 signalling [24]. The inactivation of mTORC1 leads to rapid translocation of transcription factors TFEB and TFE3 to the nucleus. Active TFEB upregulates the expression of lysosomal genes and critical regulators of autophagy, including several proteins implicated in the formation of autophagosomes and autolysosomes. Therefore, TFEB contributes to the synchronization of autophagy and lysosomes [25]. TFEB can also mediate lysosomal exocytosis and secretion of their cargo including the proteolytic enzymes, such as cathepsins, which results in extracellular matrix remodelling and invasion of cancer cells [26].

The early stage of autophagy machinery is the activation of the ULK1 (unc51-like autophagy-activating kinase 1) complex. This complex consists of ULK1, FIP200, ATG13, and ATG101 (see Fig. 3). ULK1 complex forms puncta usually associated with the endoplasmic reticulum (ER). ER membrane provides local support for many (putatively different) membrane sources and initiates the formation of the isolation membrane (commonly referred to as the phagophore) from the omegasome (a phosphatidylinositide-3-phosphate (PI3P)-enriched subdomain of the ER membrane) $[27,28]$. The omegasome serves as a cornerstone for the formation of the phagophore. To the omegasomes, ATG9 vesicles and coat protein complex II (COPII) vesicles are recruited to elongate the autophagosome. The origin of the rest of the lipid bilayers is currently unknown [29]. ATG9 migrates through the trans-Golgi network and the endosomal system under nutrient-rich conditions and transiently binds to the autophagosome in case of autophagy induction. The trafficking of ATG9 through the recycling endosomes may be a fundamental step for autophagosome genesis [30] as ATG9 meets and fuses with ATG16L1 in a VAMP3-dependent manner in recycling endosomes [31].

Once activated, ULK1 phosphorylates the class III phosphoinositide 3-kinase (PI3K) complex I (consisting of VPS34, VPS15, Beclin1, ATG14L, and NRBF2 [16]; see Fig. 3). VPS34 generates PI3P enabling the recruitment of autophagy-related PI3P-binding proteins such as WIPI and DFCP1 [32]. In some circumstances, such as shear stress, PI3KC2 $\alpha$-dependent and VPS34-independent generation of PI3P can take place [33]. Expansion of the phagophore requires the ATG2A-WIPI4 complex mediating ER-phagophore association and establishing the transfer of lipid membranes from the ER and the vesicles to the phagophore [34,35]. One of the key molecular events of autophagosome formation is the lipidation of ATG8-family proteins with phosphatidylethanolamine (PE). Mammals express 2 subfamilies of ATG8 proteins: the LC3 subfamily consisting of LC3A, LC3B, LC3B2, and LC3C (referred to here as LC3; microtubule-associated protein light chain 3 ) and the GABARAP subfamily consisting of GABARAP, GABARAPL1, and GABARAPL2 (referred to here as GABARAP). Lipidation of LC3 and GABARAP is a membrane-curvature dependent process [36] catalysed by E1-like activating enzyme ATG7, E2-like conjugating enzyme ATG3 and enhanced by the ATG12-ATG5-ATG16L1 system formed in the previous step [37, 38]. The cysteine protease ATG4B executes two LC3/GABARAP processing events: priming of newly synthesized pro-LC3/GABARAP to enable lipidation (newly translated LC3B, called pro-LC3, is cleaved by the cysteine protease ATG4B at the C-terminal section to give LC3-I) and deconjugation of lipidated LC3/ GABARAP after cargo degradation in autolysosome [39] (see Fig. 4). ATG4B is considered to be the main isoform of ATG4 as it possessed the broadest spectrum against all substrates, followed by ATG4A, whereas ATG4C and ATG4D had minimal activity [40]. The final conjugation of LC3-I to PE molecules results in the formation of membrane-bound LC3-II. LC3-II is specifically targeted to the elongating phagophore and remains on autophagosomes until their fusion with lysosomes [37]. The proper closure of the autophagosomal membrane requires the ESCRT-III component CHMP2A and the activity of VPS34 [41]. While the LC3 subfamily mediates the elongation of the phagophore, GABARAP proteins probably function in the final sealing of the autophagosome [42]. GABARAP subfamily positively regulates ULK1 activity and phagophore formation in response to starvation. On 

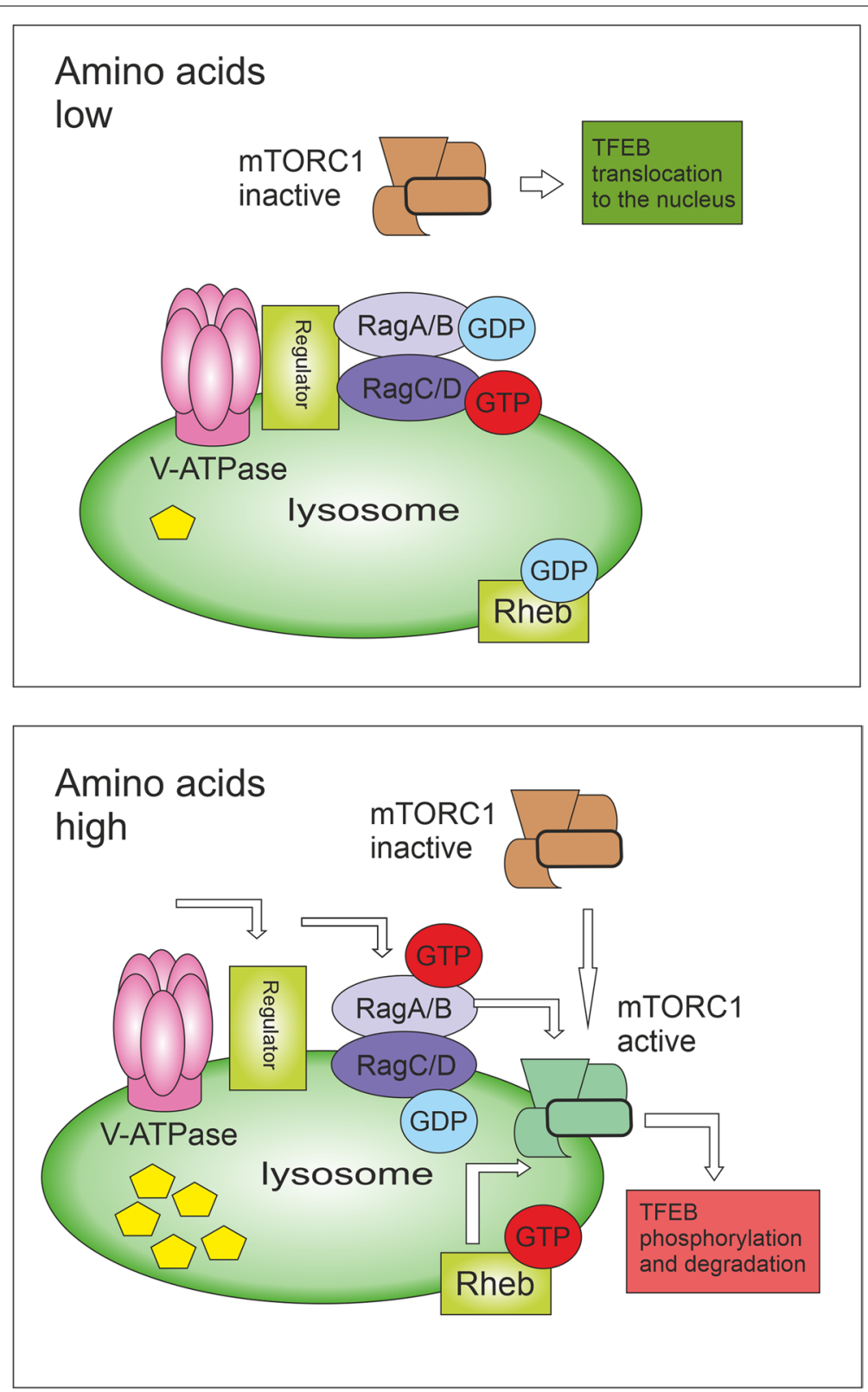

Fig. 2 Amino acid-based mTORC1 activation. By amino acid starvation, the inactive V-ATPase-Ragulator complex is unable to activate Rag GTPases on the lysosomal surface, thus mTORC1 is not recruited to the lysosome. The inactivation of mTORC1 leads to rapid translocation of transcription factors TFEB and TFE3 to the nucleus. Active TFEB upregulates the expression of lysosomal genes and critical regulators of autophagy. By amino acid abundancy, the V-ATPase undergoes conformational changes leading to the activation of Regulator, which in turn promotes the Rag heterodimer activation. Active Rag heterodimer (RagA/B(GTP)-RagC/D(GDP)) then recruits mTORC1 to the lysosomal surface where Rheb is present. Rheb can directly bind and activate mTORC1. TFEB is recruited on the lysosomal membrane, phosphorylated by active mTORC1, and then degraded by the proteasome

the other hand, the LC3 subfamily regulates them negatively [43].

Autophagosomes are transported along microtubules, which require the function of dynein. Consequently, inhibition of dynein-dependent transport or depolymerization of microtubules results in the inhibition of autophagy [44]. The degradative autophagy culminates in a fusion of closed autophagosomes with lysosomes where the cargo is eventually degraded. The autophagosome-lysosome fusion is managed by Syntaxin-17 (STX17) on autophagosomes (see Fig. 4), which binds the VAMP8 (vesicle-associated membrane protein 8) on 
the lysosomal membrane via the Qbc-SNARE SNAP29 (synaptosome-associated protein 29). Accessory proteins such as ATG14 and homotypic fusion, protein sorting (HOPS) tethering complex, ESCRT, RAB7, and the class $C$ VPS proteins are also needed $[45,46]$. Some studies indicate that the kinase ULK1 regulates STX17 engagement during autophagosome maturation. Unphosphorylated ULK1 recruits STX17 and increases its affinity towards SNAP29. Protein kinase C alpha (PKC $\alpha$ ) mediated phosphorylation of ULK1 does not change its kinase activity but decreases autophagosome-lysosome fusion $[47,48]$. Inactivation of mTORC1 might also be required to facilitate the fusion between autophagosomes and lysosomes [49]. In addition to managing autophagy induction in complex I, complex VPS34-Beclin1 has a role in the fusion of autophagosomes with lysosomes as complex II (see Fig. 3). UVRAG competes with ATG14L for binding to Beclin1 [50]. When bound to Beclin1, UVRAG stimulates RAB7 GTPase activity and autophagosome fusion with lysosomes or multivesicular bodies (late endosomes; MVBs) [51]. VPS34-Beclin1-UVRAG complex may also contribute to autophagy induction via Bif-1/Endophilin B1-mediated activation of VPS34 [52].

\section{Autophagy in the progression and therapy of solid cancers}

Autophagy is undoubtedly an important tumour suppressive mechanism maintaining cellular homeostasis by executing lysosomal degradation of toxic material in the cell, as well as mediating intercellular communication via proteins and hormones with signalling function that can be secreted in an autophagy-mediated manner [53]. During the early phases of cancerogenesis, autophagy has significant cytoprotective and tumour-suppressive potential. Dysfunction of this process is associated with an increased risk of cancer development. Haploinsufficiency of the $B E C N 1$ gene was observed in $40-75 \%$ of sporadic human breast and ovarian cancers $[54,55]$ and more than $25 \%$ of gastric and colorectal tumours are haploinsufficient in one of the $A T G 2 B, A T G 5, A T G 9 B$, or ATG12 genes [56]. In multiple cancer types, ATG5 mutations and alternative mRNA splicing disrupt the ATG16L1-binding to ATG5 and impair the ATG12-ATG5 conjugation. Furthermore, ATG16L2 is overexpressed in several cancers and competes with ATG16L1 for binding to ATG5 resulting in proteasomal degradation of ATG16L1 and disruption of autophagy [57]. The tumour-suppressive effect of autophagy is also supported by the fact that autophagy is stimulated by some tumour suppressors, including PTEN, TSC, or DEPTOR [58-61] (Role of autophagyrelated proteins in solid cancers is summarized in Table 1). Nevertheless, if tumourigenesis has been started up, autophagy can further support tumour progression. Many aggressive tumours need autophagy for important tumour-promoting processes (e.g. autophagy enables ERBB2 (Erb-B2 Receptor Tyrosine Kinase 2) trafficking and supports tumourigenesis in ERBB2-driven breast cancer [111]). Increased autophagic activity mediates an escape of premalignant cells from genotoxic stress or anoikis, suppresses immune surveillance and can result in intrinsic resistance against anticancer therapy [112114]. Autophagy also increases the metabolic plasticity of tumour cells, allowing them to survive in adverse conditions and supports forming of cancer stem cells [115].

Because the induction of autophagy has been observed as a side effect of many cytotoxic anticancer therapies causing therapy resistance, a large number of strategies using autophagy inhibition have been proposed to increase the efficacy of these therapies [116]. Inhibition of autophagy in the tumour microenvironment can disrupt metabolic communication between tumour and stromal cells [117] and decrease

\footnotetext{
(See figure on next page.)

Fig. 3 Macroautophagy pathways. The autophagic process is divided into five stages including initiation, phagophore nucleation, phagophore formation, autophagosome-lysosome fusion, and cargo degradation in autolysosomes. Signals activating macroautophagy usually originate from starvation, hypoxia, oxidative stress, and stress of the endoplasmic reticulum (ER). These signals trigger the activity of Unc-51-like kinase 1 (ULK1) complex (consisting of ULK1, FIP200, ATG13, and ATG101), which then starts phosphorylation of components of the class III PI3K (PI3KC3) complex I (consisting of VPS34, VPS15, Beclin1, ATG14L, and NRBF2) enabling nucleation of the phagophore. VPS34 produces phosphatidylinositol-3-phosphate (PI3P) allowing the recruitment of autophagy-associated PI3P-binding proteins such as DFCP1 and WIPI mediating the initial stages of autophagosome formation by associating ATG2A stably to PI3P-containing areas. Expansion of the phagophore requires the ATG2A-WIPI complex mediating ER-phagophore association and establishing the transfer of lipid membranes from the ER and the vesicles to the phagophore. WIPI was also shown to bind ATG16L1, thus recruiting the ATG12-ATG5-ATG16L1 complex. Elongation of autophagosomes requires the ubiquitin-like conjugation system managing the orchestrated activity of ATG proteins and LC3 (microtubule-associated protein light chain 3) and/or GABARAP. The ATG12-ATG5-ATG16L1 complex enhances the final connection of phosphatidylethanolamine (PE) molecules resulting in the formation of membrane-bound LC3-II and/or GABARAP-PE. Cellular membranes, including the mitochondrial membrane, the plasma membrane, recycling endosomes, and the Golgi complex, contribute to the elongation of the phagophore by providing membrane material. Elongation of the phagophore gives rise to double-layered vesicles called autophagosomes. In addition to managing autophagy induction in complex I, complex VPS34-Beclin1 has also a role in the fusion of autophagosomes with lysosomes as complex II. UVRAG competes with ATG14L for binding to Beclin1. When bound to Beclin1, UVRAG stimulates RAB7 GTPase activity and autophagosome fusion with lysosomes. Autophagosome-lysosome fusion is managed by Syntaxin-17 (STX17) on autophagosomes, VAMP8 on lysosomes, and by accessory proteins such as ATG14 and homotypic fusion, and protein sorting (HOPS) tethering complex
} 


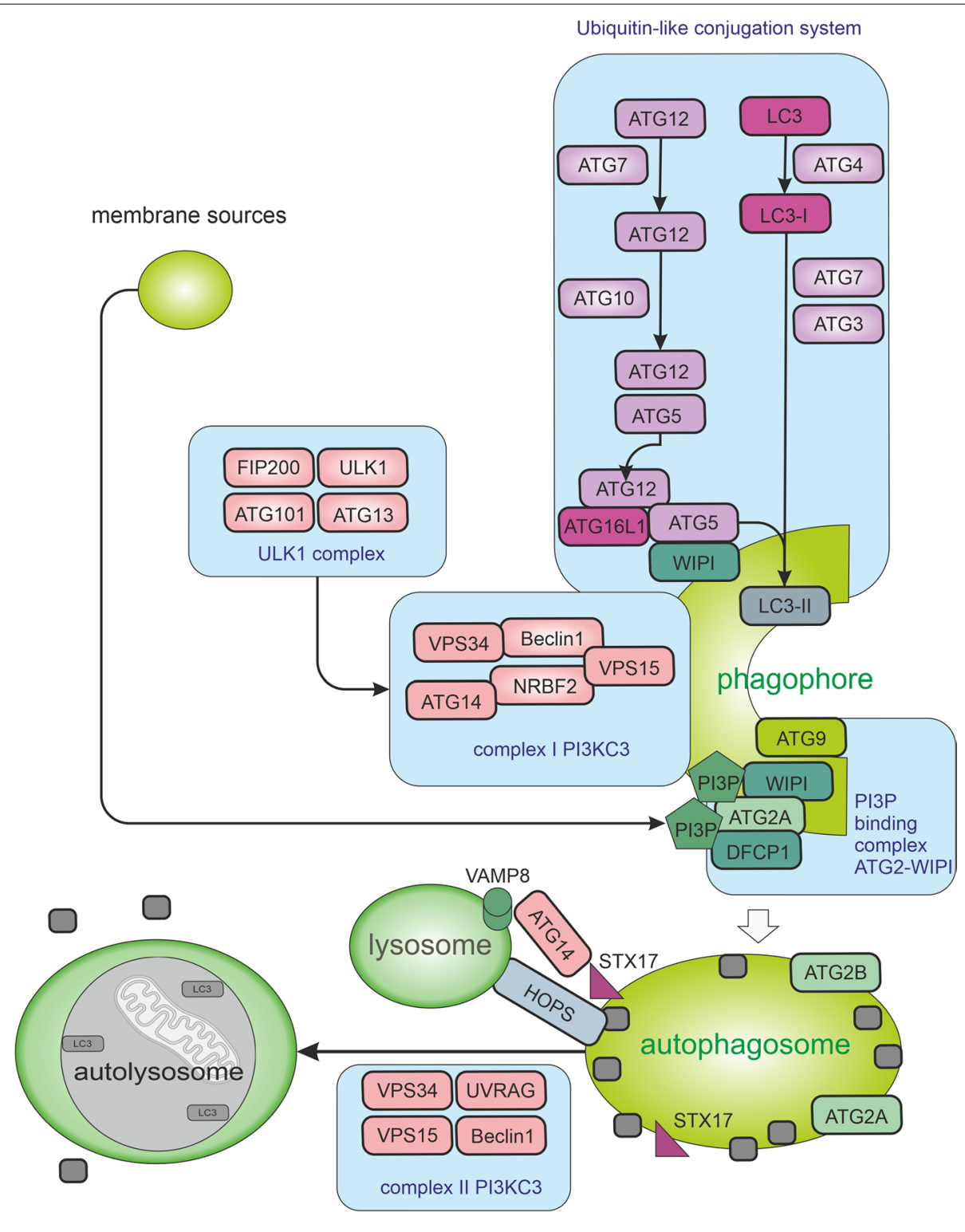

LC3-II and/or GABARAP

Fig. 3 (See legend on previous page.)

cell motility of metastatic tumour cells as inhibition of autophagy reduces disassembly of focal adhesions at the leading edge of the cell [118]. Systemic acute deletion of ATG7 in adult mice with preexisting non-small cell lung cancer reversed lung adenocarcinomas initiated by the KRASG12D oncogenic mutation and p53 deficiency to a benign form of tumour (oncocytomas) and blocked cell proliferation and cancer cell survival [119]. Furthermore, loss of tuberous sclerosis complex 2 (TSC2) sensitizes cancer cells to nelfinavir-bortezomib therapy due to intensifying endoplasmic reticulum stress-induced cell death [61]. On the other hand, autophagy induction causes a decrease in the levels of transcription factors triggering EMT [120], reduces 6-phosphofructo-2-kinase/fructose-2,6-biphosphatase 3 (Pfkfb3) expression and elicits metastatic dormancy in breast cancer stem cells [121]. Consequently, many studies are demonstrating the benefit of autophagy during cancer therapies, especially in inducing immunogenic cell death. Tumour cells dying of autophagic 


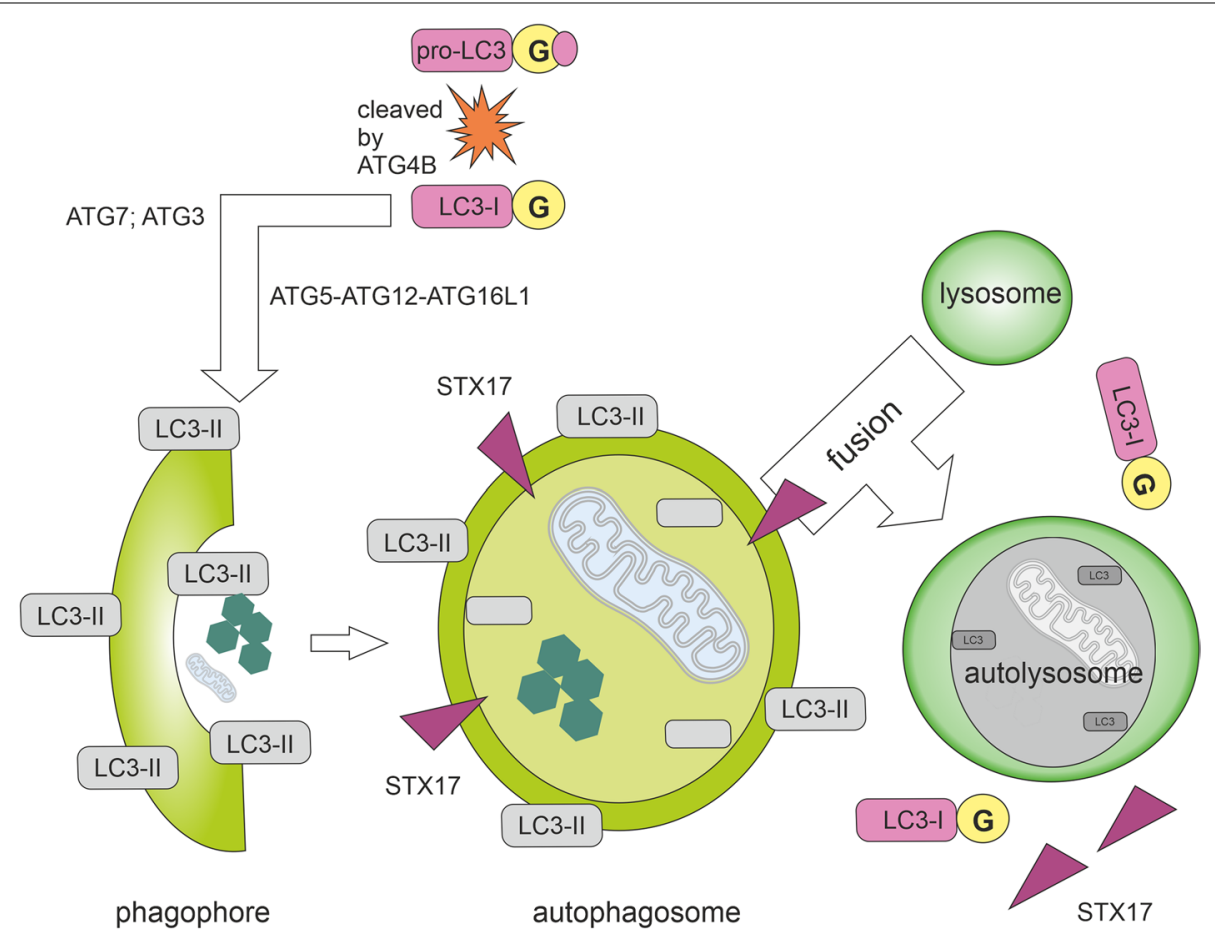

Fig. 4 Autophagy with emphasis on the state of MAP1LC3B (LC3B). Newly translated LC3B, called pro-LC3, is cleaved by the cysteine protease ATG4B at the C-terminal end with subsequent exposure of glycine residues (G). A cleaved form of a protein (LC3-l; soluble LC3) is further processed by ATG7 and ATG3, which conjugates LC3-I to phosphatidylethanolamine (PE) molecules. The ATG12-ATG5-ATG16L1 complex enhances the final conjugation of LC3-I to PE molecules resulting in the formation of membrane-bound LC3-II specifically targeted to the elongating autophagosomal membrane. The two ends of the insulating membrane are subsequently joined together to form an autophagosome with a double membrane. STX17, which is located on the outer autophagosomal membrane (not on the isolation or lysosomal membrane), is required for the fusion of an autophagosome with a lysosome. In the resulting autolysosome, the material is cleaved by acid hydrolases. During internal degradation, STX17 proteins are released from the outer membrane of the autophagosome. LC3-II molecules conjugated to the inner autophagosomal membrane are degraded by acids hydrolases, while the LC3-II molecules on the outer membrane of the autophagosome are cleaved by ATG4B and recycled as LC3-I

cell death leads to the recruitment of immune cells to the tumour site and the activation of a tumour-specific immune response. Accordingly, caloric restriction (which promotes autophagy by inactivating mTORC1) has resulted in increased control of the immune system over the tumour, but only in tumours capable of autophagy [122].

Autophagy may be one of the key modulators of the tumour microenvironment (TME). The autophagydependent secreted soluble factors or factors contained in EVs may enable metabolic manipulation of non-cancer cells in the tumour microenvironment, stimulate cellular proliferation, invasive phenotype, and promote immunosuppression [53]. This TME-modulating theory is supported by the fact that autophagy facilitates the selection of material for unconventional secretion of certain cytoplasmic proteins [123]. Intact autophagy machinery is required for the secretion of multiple factors favouring invasion, including interleukin-6 (IL-6), MMP2, and WNT5a [124]. Autophagy is also closely related to the biogenesis and secretion of exosomes and amphisomes.

\section{Autophagy and MVBs}

Autophagy and endocytic pathways are important in managing many aspects of homeostasis as both endosomes and autophagosomes are known to deliver cellular material to lysosomes for degradation. Autophagy and exocytosis seem to be largely interconnected as autophagy cargo can be released by amphisomes derived from multivesicular bodies (MVBs) and phosphoinositide-3-phosphate (PI3P) is essential for the genesis of both endosomes and autophagosomes, and their positioning as PI3P promotes the microtubule-dependent translocation of late endosomes and lysosomes to the cell periphery. This PI3P-dependent lysosome translocation to the cell periphery promotes mTORC1 activation [125]. The most of cellular PI3P is generated by class III PI3K VPS34 in complex II with a small contribution of class II PI3Ks [126]. Binding and activation of VPS34 


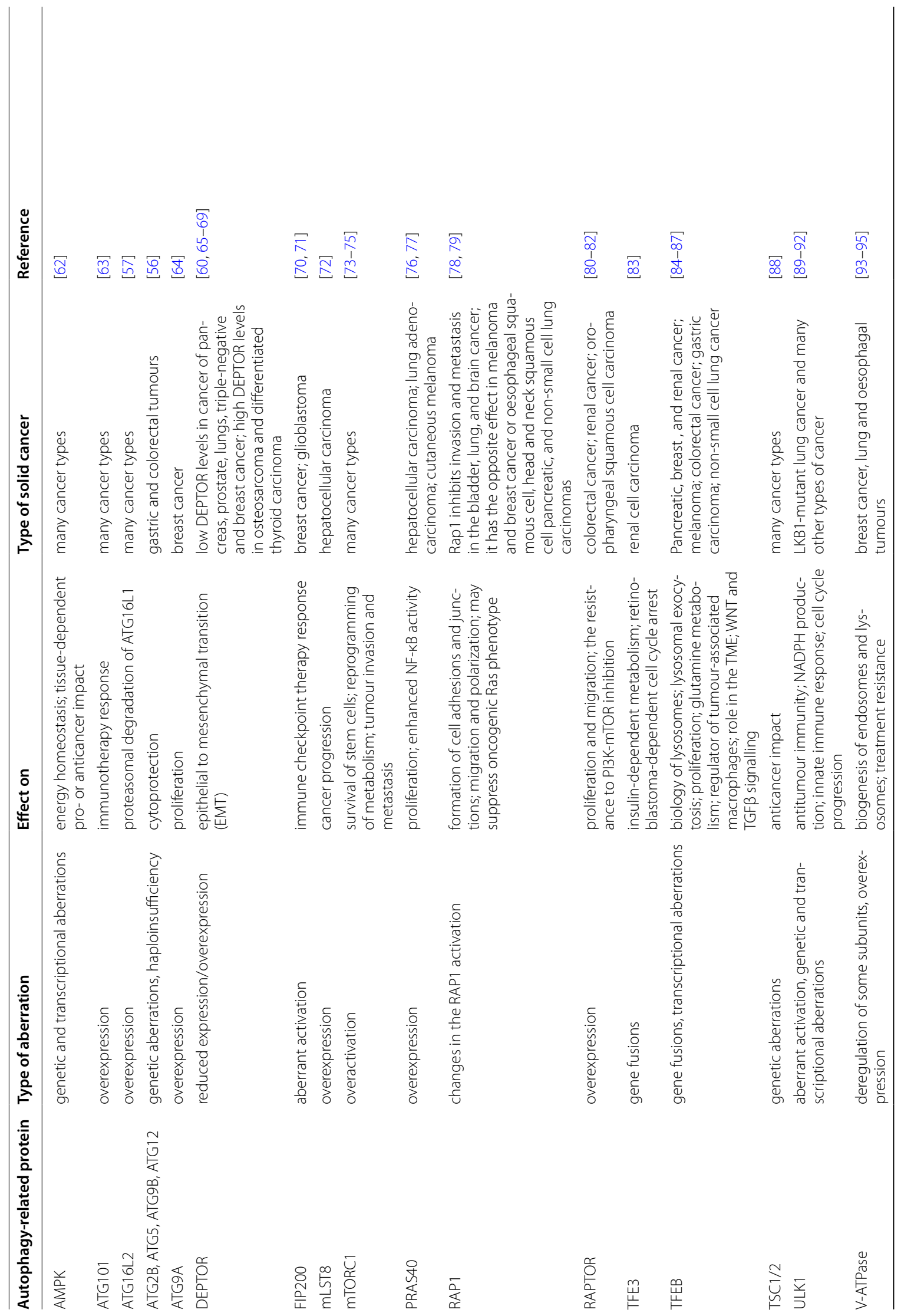




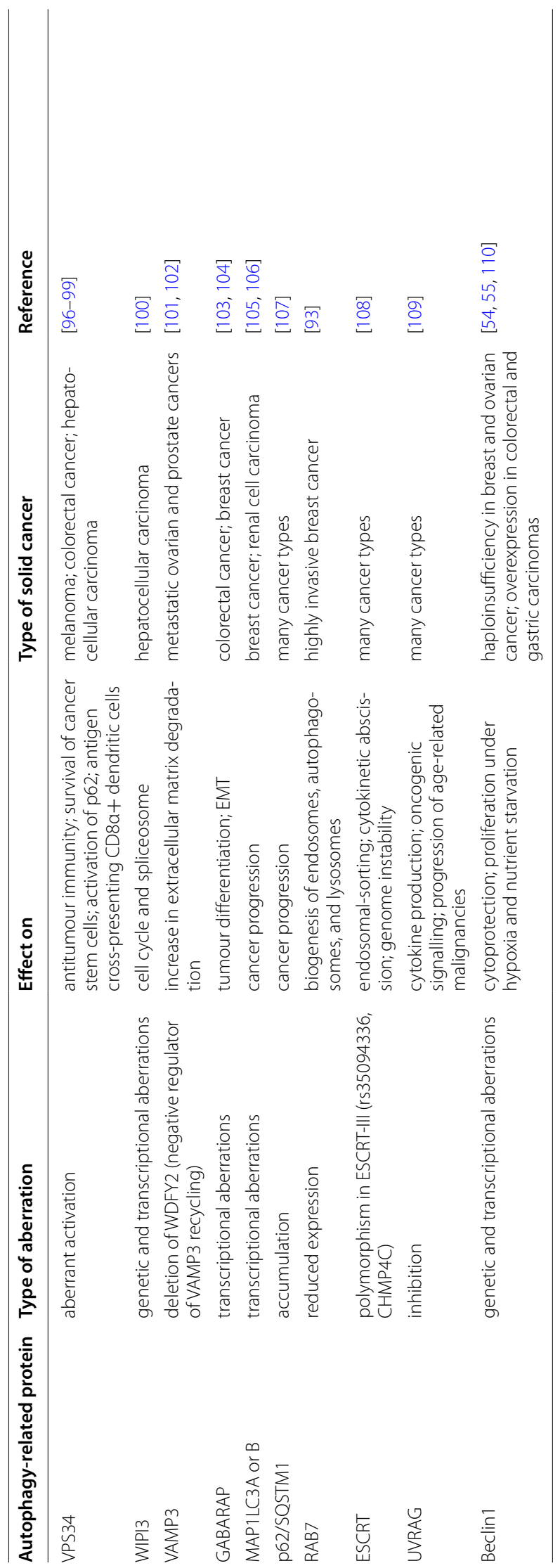


on endosomes are initiated through the recruitment of RAB5 to endosomes by the guanine nucleotide exchange factor Rabex5 (RAB Guanine Nucleotide Exchange Factor 1) [127]. VPS34 then produces PI3P increasing the binding of RAB5 and other downstream effectors, including early endosome autoantigen 1 (EEA1), the hepatocyte growth factor-regulated tyrosine kinase substrate (HRS; ESCRT-0 subunit) regulating MVBs formation via ESCRT recruitment to endosomes [128]), and endosomal sorting nexin protein family (SNX) [129]. Overexpression of SNX3 may alter the morphology of endosomes and delay their transport to the lysosome [130]. SNX18 was identified as a positive regulator of autophagosome formation [131]. Consequently, VPS34 plays a crucial role in endosome biogenesis through EAA1 and other RAB5 effectors, vesicle invagination and cargo selection within MVBs, and the fusion of autophagosomes with lysosomes. Inhibition of VPS34 resulted in dysfunction in autophagy, vesicular trafficking, and endocytic recycling and sorting [132, 133]. Furthermore, proteins such as Beclin1 and ATG14L that regulate PI3P levels are positive modulators of autophagy [134]. Some data suggest that surface delivery of endosomal cargo requires hydrolysis of PI3P mediated by MTM1 as the endosomal accumulation of PI3P inhibits exocytosis. Defects caused by mutations in MTM1 can be partially reversed by pharmacological inhibition of VPS34 [135].

Endosomal maturation is accompanied by conversion from early endosomal RAB5 to late endosomal RAB7 and active cargo sorting into intraluminal vesicles (ILVs) by the ESCRT complex. The transition from early to late endosomes is complicated by a positive feedback loop between Rabex 5 and RAB5. It was demonstrated that SAND1/MON1A is needed to interrupt this positive feedback loop by displacing Rabex 5 from endosomal membranes. SAND1/MON1A also manages the recruitment of RAB7 (see Fig. 5) [127]. Then VPS34 recruits TBC1D2 protein to endosomes in a RAB7-dependent manner to further inactivate RAB5 and to facilitate early to late endosome maturation. VPS34 inhibition causes hyperactivation of RAB7, autolysosomal dysfunction, a phenotype with large late endosomes and an enhanced release of atypical exosomes harbouring poly-ubiquitinated proteins [136-138]. Interestingly, RAB7 can participate in both MVBs degradation and/or MVBs-related secretion as it regulates autolysosome maturation and simultaneously the secretion of syntenin and syndecancontaining exosomes [139]. RAB7-associated endosomal processes depend not only on RAB7 GTP-based state but also on modifications with ubiquitin [140]. Endosomal maturation during the late endosome/lysosome pathway is accompanied by conversion of PI3P to $\mathrm{PI}(3,5) \mathrm{P}_{2}$ at the limiting membrane of late endosomes. This process depends on PIKfyve (phosphoinositide kinase, FYVEtype zinc finger containing). Consequently, the activity of PIKfyve is vital for the sorting of cargo into MVBs [141].

In addition to the role in the degradation and recycling of cellular waste, autophagic and endo-lysosomal systems can play a key role in secretory pathways (see Fig. 6) as autophagy cargo can be released by amphisomes derived from multivesicular bodies (MVBs). MVBs are late endosomes containing many intraluminal vesicles (ILVs) formed by the invagination of the endosomal membrane. ILVs start to be generated in early endosomes and accumulate until the late endosomal stage. In vitro budding of ILVs into MVBs is regulated by syntenin-syndecan interactions requiring Alix which is known to interact with several ESCRT proteins including TSG101 and CHMP4 [142, 143]. MVBs can fuse with the plasma membrane to release intraluminal vesicles (ILVs) to the extracellular space as exosomes. During exosome biogenesis, Alix forms a complex with the scaffold protein syntenin, mediating the loading of cargo into exosomes and promoting exosome release. These Alix-dependent processes are controlled by ATG12-ATG3 and cells lacking ATG12ATG3 showed reduced exosome biogenesis. Both ATG12-ATG3 and Alix promote basal, but not starvation-induced, autophagic flux [9]. Some results indicate that activated c-Src in the endosomal membrane promotes the secretion of exosomes. Alix was identified as a c-Src-interacting protein in exosomes, resulting in the upregulation of exosome secretion in c-Src-transformed cells [143]. The small GTPase ADP ribosylation factor 6 (Arf6) and its effector phospholipase D2 (PLD2) also regulate the syntenin pathway [144]. MVBs morphology and their docking to the plasma membrane is significantly disrupted by the loss of RAB27a and/ or RAB27b activity [145]. Critical plasma membrane docking and secretion sites for MVBs are invasive actin structures called invadopodia. Invadopodia degrade the extracellular matrix through the local deposition of matrix metalloproteinases (MMPs) and manage cancer cell invasion [146]. Protein WDFY2 (WD Repeat and FYVE Domain Containing 2) is frequently lost in metastatic cancers (e.g. ovarian and prostate cancers). Through its interaction with VAMP3, WDFY2 restricts the budding of MMP14-containing VAMP3 vesicles from actin-stabilized endosomal tubules. Upon deletion of WDFY2, this negative control is disrupted and faster recycling of MMP14 to the plasma membrane leads to increased matrix degradation and cell invasion [102]. Furthermore, long non-coding RNA HOTAIR promotes the colocalization of VAMP3 with SNAP23 leading to MVBs fusion with the plasma membrane and exosome secretion in hepatocellular carcinoma [147]. 


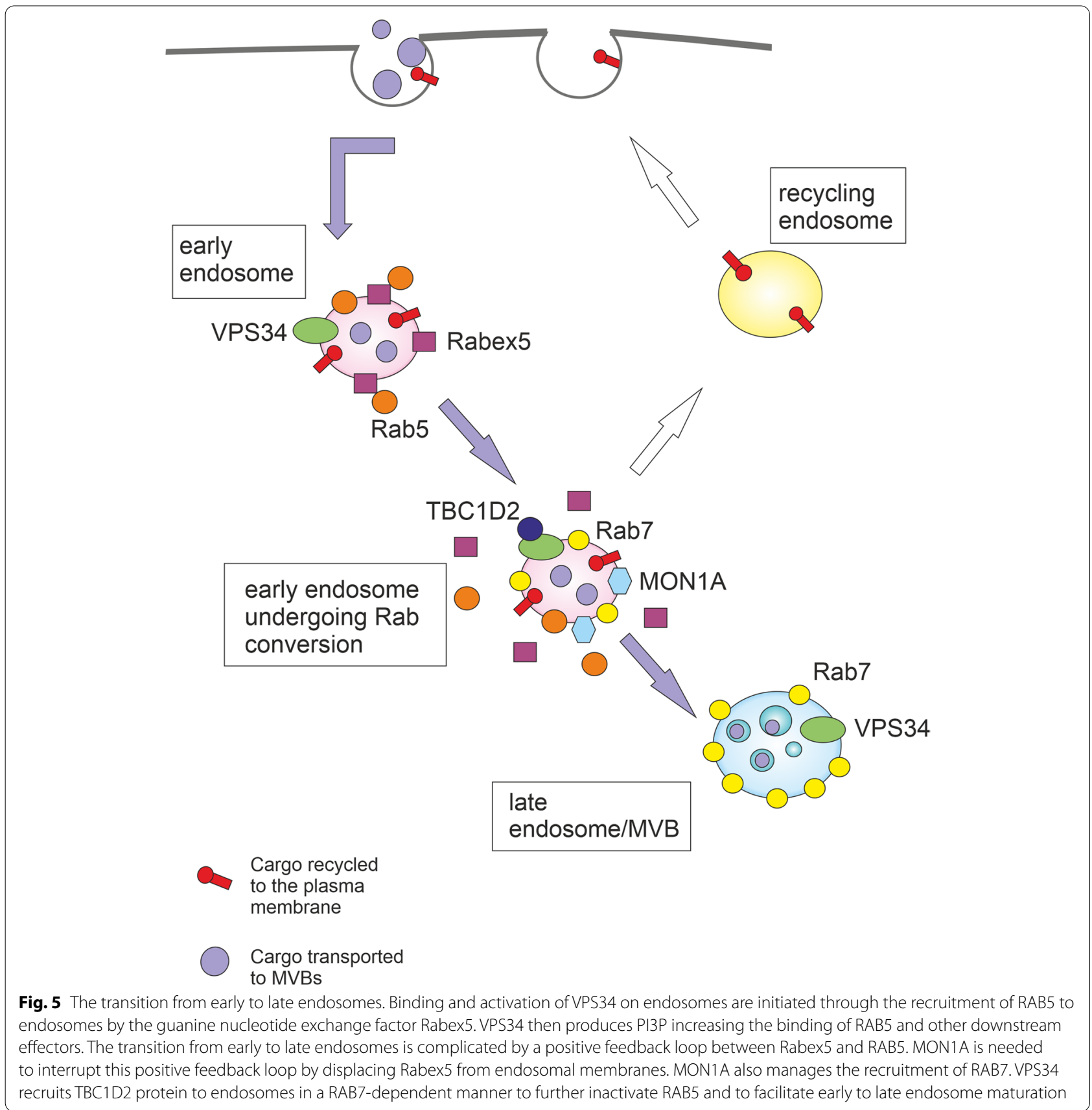

The HOTAIR also activates autophagy by upregulating ATG3 and ATG7 [148]. Long intergenic noncoding RNA 00511 (LINC00511) promotes exosome secretion by regulation of the expression of RAB27B and the colocalization of VAMP7 and SNAP23, which are involved in MVBs trafficking and their fusion with the plasma membrane [149]. LINC00511 is highly expressed in diverse cancers and correlates with poor clinical outcomes [150]. LINC00511 knockdown suppressed proliferation, invasion and autophagy in trophoblast cells
[151]. Exosome secretion was also promoted by cortactin through stabilizing cortical actin-rich MVBs docking sites [152].

Nevertheless, in healthy cells, the majority of MVBs fuse with the lysosomes, resulting in degradation of their content. ISGylation of the MVB protein TSG101 by ISG15 (interferon- $\alpha / \beta$-induced ubiquitin-like protein) induces its aggregation and degradation, being sufficient to impair exosome secretion. The secretion of exosomes is recovered when the fusion of MVBs 


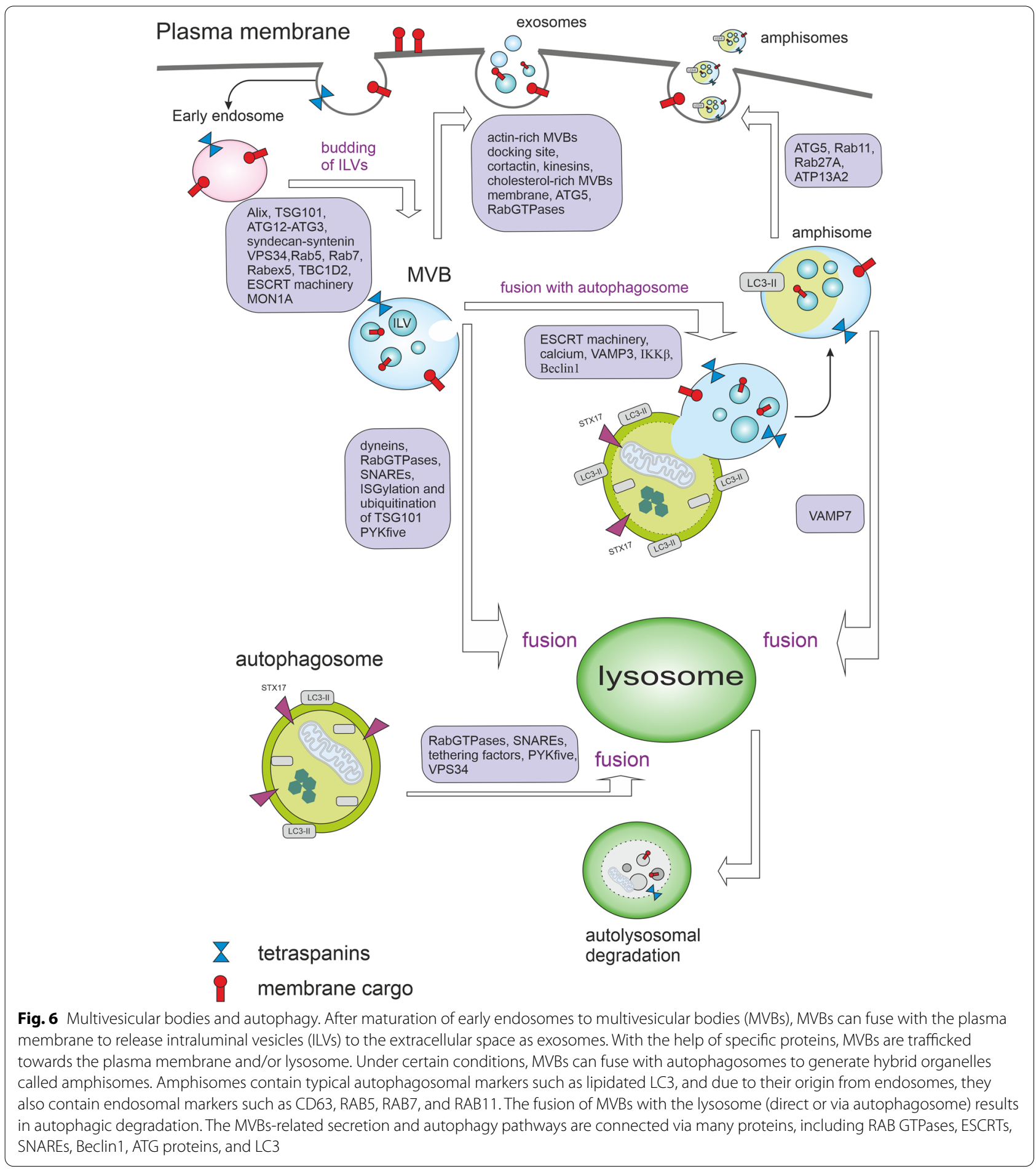

with lysosomes or autophagosomes is inhibited, indicating that the inhibition of exosome secretion is mainly mediated by the induction of MVBs degradation by the lysosomes. ISGylation reduces the number of MVBs but does not inhibit the formation of ILVs. It also promotes selective autophagy and degradation of MVBs without inducing a global autophagy response [153]. The fusion of MVBs with lysosomes can be also triggered via the ubiquitination of TSG101 by MGRN1 [154]. 
Interestingly, ubiquitination of the cargo proteins can also profoundly influence the fate of MVBs. While MVBs containing ubiquitinated major histocompatibility complex (MHC-II) underwent lysosomal degradation in immature dendritic cells, non-ubiquitinated MHC-II placed into CD9-bearing MVBs was targeted for plasma membrane fusion and secretion in activated dendritic cells. Sorting of MHC II into exosomes correlated with its incorporation into CD9 containing detergent-resistant membranes [155]. Exosomes are probably preferentially secreted from cholesterol-rich detergent-resistant MVBs whereas the cholesterol-poor MVBs are subjected to degradation [156, 157]. The alternative fate of MVBs can be also governed by their connection to dynein or kinesin. Connection to dynein motor protein (minus end-directed transport) leads to perinuclear accumulation of MVBs and lysosomal degradation of cargo by recruitment of HOPS complex. By contrast, connection to kinesin (plus end-directed transport) causes accumulation of MVBs at cell periphery $[158,159]$.

Different autophagic effectors were shown to influence the biogenesis of extracellular vesicles and their secretion. Exosome production is strongly reduced in cells lacking ATG5 and ATG16L1 as these proteins protect MVBs from lysosomal degradation and direct them into the secretory pathway. ATG5 detaches V1V0-ATPase (vacuolar proton pump) from the MVBs via LC3 which specifically decreases acidification of MVBs lumen [160]. Emerging exosomes were strongly enriched in LC3II (versus LC3-I) compared with the ratio of LC3-II to LC3-I in the corresponding cell lysates. This suggests that ATG5 and LC3 are sorted into these exosomes [160]. On the other hand, overexpression of LC3 or autophagy inducers such as starvation or rapamycin caused an enlargement of the vacuoles decorated with RAB11 and their colocalization with LC3. This situation led to the inhibition of exosome release. Even though RAB11 activity stimulates MVBs genesis and exosome release [161], the stimulatory effect of RAB11 in exosome secretion was nullified by overexpression of the autophagic protein LC3 [10].

Under certain conditions (such as shear stress [162]), autophagosomes fuse with MVBs to generate hybrid organelles called amphisomes [13]. Amphisomes contain typical autophagosomal markers such as lipidated LC3, and due to their origin from endosomes, they also contain endosomal markers such as CD63, RAB5, RAB7, and RAB11 [163]. Maturation of MVBs by ESCRT machinery is required for their fusion with autophagosomes [164] and the fusion of MVBs with the autophagosome compartment seems to be calcium- and VAMP3-dependent event $[10,163]$. The resulting amphisomes are either degraded by fusion with lysosomes or released from the cell $[162,165]$. ATG9 is required to form intraluminal vesicles in amphisomes and/or autolysosomes and is also needed for local acidification within amphisomes/ autolysosomes [166]. The fusion between amphisomes and lysosomes requires VAMP7 but not VAMP3 [163]. VAMP7 is also needed for the homotypic fusion of ATG16L1 precursors, which is a key event in the early phases of autophagy enabling membrane acquisition and autophagosome biogenesis [167]. VAMP7-labelled vesicles can be loaded with ATP and starvation triggers the delivery of the ATP-containing amphisomes toward the plasma membrane, their release, and the import of ATP to the extracellular space [168].

Amphisomes, but not exosomes, were shown to be vehicles for the active release of DNA from the cell [169]. The formation of an amphisome may negatively regulate the coordination between exosome secretion and autophagy. For example, rapamycin or starvation promoted MVBs-autophagosome fusion and reduced exosome secretion in the K562 cell line [10]. On the other hand, the exosome release of toxic/damaged material may provide a cellular mechanism bypassing the autophagic defects caused by ageing or different pathological states [170].

Amphisomes can participate in immune response as they can function as anti-viral machinery by sequestering and exporting viral proteins from the cell [171]. IFNY is a cytokine critical for innate and adaptive immunity against viral infections. Upon IFN $\gamma$-induced autophagy in lung epithelial cells, amphisomes containing annexin 2 (ANXA2) were released. This process was dependent on ATG5, RAB11, and RAB27A [165].

Interleukins IL-1 $\beta$ and IL-18 are potent pro-inflammatory cytokines crucial for responses to infection and injury. Secretion levels of these cytokines increased when low-autophagy cells were treated with the autophagyinducing tat-Beclin1 peptide and decreased when ATG7 was silenced in high-autophagy cells [172]. An unobstructed autophagy pathway and functional MVBs are necessary for inflammasome-dependent IL- $1 \beta$ and IL-18 secretion [173, 174]. Nevertheless, it is not completely clear if IL- $1 \beta$ is secreted by amphisomes or by modified autophagosomes. Similarly to the degradative autophagosomes, modified secretory autophagosomes have a double membrane decorated with LC3-II. In degradative autophagosomes, STX17 manages the fusion with the lysosome. However, in secretory autophagosomes, SEC22B in combination with plasma membrane syntaxin 3 and syntaxin 4 as well as SNAP23 and SNAP29 facilitate fusion with the plasma membrane and cargo secretion $[175,176]$. Autophagy-dependent secretion of IL-1 $\beta$, IL-6, CSF3/G-CSF, CXCL1, TREM1, CCL2, CCL3/ MIP-1 $\alpha$, and CXCL2 in response to UVB radiation was 
also described. Secretion of these cytokines was blocked by conditional ATG7 depletion [177].

Another factor interconnecting cell stress and immune response with autophagy and amphisome genesis is IкB kinase $(\mathrm{IKK} \beta)$. IKK $\beta$ is the predominant catalytic subunit of the IKK complex and is required for the activation of the canonical NF- $\mathrm{KB}$ signalling pathways. IKK $\beta$ activation also induces the fusion of MVBs with autophagosomes to form amphisomes and promotes their secretion in tumour cells. This secretion was absent or strongly reduced when autophagosome formation was impaired by 3-methyladenine or ATG7 inactivation [178]. In breast cancer cells independent of autophagy, ATG7 inhibition by shRNAs increased IL- 6 secretion. On the other hand, in autophagy-dependent cells, ATG7 inhibition decreased IL-6 secretion, cell survival and mammosphere formation [179]. Besides RNA interference-mediated ATG7 depletion, pyrazolopyrimidine sulfamate compounds were found to be potent selective inhibitors of ATG7 [180].

In cancer cells, selective and non-selective autophagy and EVs secretion are often amplified because of harsh conditions in TME, such as hypoxia or ER stress [181183]. Some regulators such as GAIP interacting protein $C$ terminus (GIPC) control both EVs and autophagy pathways [184]. GIPC knockdown led to significant inhibition of pancreatic carcinoma growth in an orthotopic mouse model [185]. The crosstalk between endosome-related secretory pathways and autophagy orchestrates the intratumoural communication as autophagy significantly impacts not only the quantity of EVs but also their content. On the other hand, EVs can significantly influence the dynamic of autophagy in TME [186, 187]. Exosomes derived from breast cancer cells stimulate beige/brown differentiation and reprogram metabolism in stromal adipocytes to promote cancer progression [187]. It was also shown that hypoxic glioma-derived exosomes promote M2-like macrophage polarization by enhancing autophagy induction [188] and MCF-7 breast cancer cells with undetectable MMP2 protein acquired expression of MMP2 and corresponding gelatinase activity after stimulation with exosomes derived from mesenchymal stromal stem cells [189].

\section{Autophagy modulators and their effect on EVs secretion}

Autophagy is a multi-step process. Each step can potentially be inhibited. Progress within the field has led to the development of agents targeting almost all phases of this process (see Fig. 7). Targeting the specific stage of autophagy may profoundly influence resulting secretory pathways as the early-stage autophagy inhibition does not seem to be equivalent to the late-stage inhibition.
Furthermore, one compound (such as tacrine-melatonin heterodimer C10) can induce the early stages of autophagy and inhibit it at the late stages. Transitory treatment by $\mathrm{C} 10$ induced secretion of proinflammatory cytokine IL-6, proving interconnection between autophagy and secretory pathway [190]. Some amount of IL-6 produced was found to be secreted by exosomes [191, 192]. On the other hand, IL-6 inhibits starvation-induced autophagy and activates stress-induced autophagy via the STAT3 signalling pathway [193, 194].

\section{ULK1 inhibitors}

ULK1 is a serine/threonine-protein kinase. In most cell lines, loss of ULK1 is sufficient to disrupt autophagy [196]. However, ULK2 acts with a degree of redundancy with ULK1 [197]. MRT68921 potently inhibits both ULK1 and ULK2. ULK1 inhibition results in the accumulation of immature early autophagosomal structures, indicating a role for ULK1 in the initiation and maturation of autophagosomes [198]. ULK1 activity also manages the trafficking of ATG9 [199] which is required for intraluminal vesicle formation within amphisomes and autolysosomes [166]. Consequently, inhibition of ATG9 causes a reduced capacity to degrade endosomal cargo, which may result in enhanced EVs secretion. Amino acid starvation or rapamycin causes a redistribution of ATG9 from the trans-Golgi network to peripheral, endosomal membranes. The redistribution of ATG9 requires PI3K activity and is reversed after the restoration of amino acids [199]. On the other hand, AMPK-ULK1-mediated but mTOR-independent signalling plays an important role in the induction of autophagy-mediated PARK7 secretion. 6-hydroxydopamine-induced oxidative stress triggered PARK7 secretion which was suppressed by cotreatment with MRT68921 [200]. Nevertheless, PARK7 seems not to be secreted by classical exosomes [169]. MRT68921 can also disrupt the signals between lysosomes and autophagic machinery. The lysosomal calcium channel TRPML1 connects lysosomal calcium to autophagosome biogenesis through the triggering of the CaMKK $\beta /$ VPS34 pathway. TRPML1-mediated generation of PI3P requires functional VPS34 and ULK1 [201]. RNAi-mediated knockdown of ULK1 and/or ULK2 resulted in impaired endocytosis of nerve growth factor (NGF) [202]. MRT68921 was also shown to be a potent inhibitor of NUAK1 (NUAK family SNF1-like kinase 1) which is a critical component of the antioxidant defence necessary for the survival of tumour cells during cytotoxic therapy and EMT. As cytotoxic therapy induces elevated ROS levels and triggers the ULK1 pathway to activate protective autophagy and mitophagy, dual targeting of NUAK1 and ULK1 by MRT68921 can be beneficial in tumour management [203]. ULK1 inhibition also 


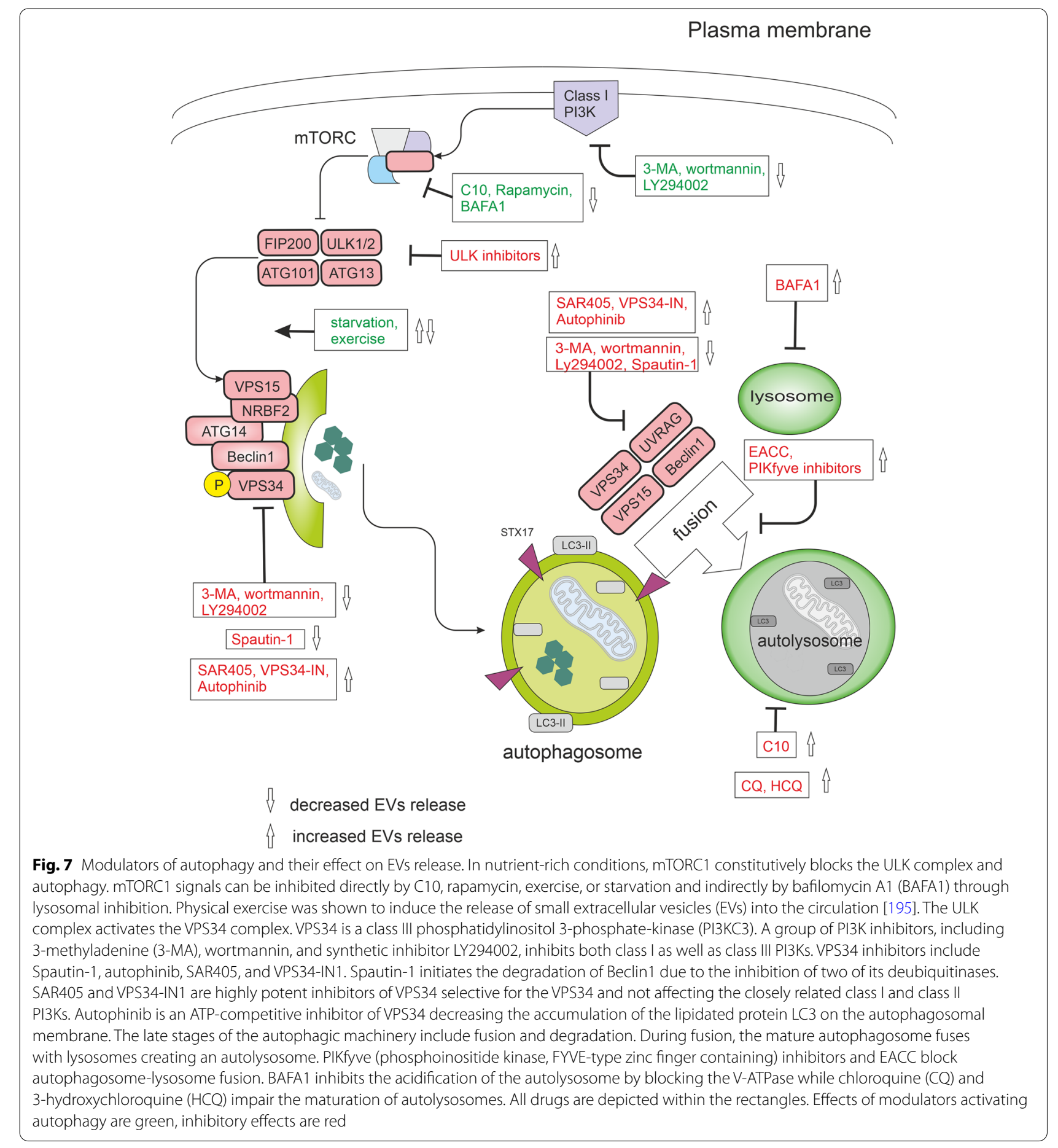

overcomes compromised antigen presentation in LKB1 (liver kinase B1)-mutant lung cancer [89].

\section{Pan-PI3K inhibitors}

Phosphoinositide 3-kinases (PI3Ks) are divided into three classes: class I, class II, and class III. Class I PI3Ks predominantly produce $\mathrm{PI}(3,4,5) \mathrm{P} 3$ (and indirectly, PI $(3,4)$ P2), class III PI3Ks synthesise PI3P, and to a lesser extent, class II PI3Ks synthesise PI3P and PI $(3,4)$ P2). PI $(3,4) \mathrm{P} 2$ was described as a key mediator of the late stages of clathrin-mediated endocytosis [126]. $\mathrm{PI}(3,4,5) \mathrm{P} 3$ at the plasma membrane recruits effectors 
such as the protein kinase B/Akt. Active Akt controls many downstream pathways such as the mTORC1 pathway. Activated mTORC1 destabilizes the ULK1 complex and inhibits autophagy [204]. The role of PI3P has been discussed above.

A group of PI3K inhibitors includes 3-methyladenine (3-MA), wortmannin, and synthetic inhibitor LY294002. These compounds inhibit class I PI3Ks as well as class III PI3Ks. Wortmannin and 3-MA have been routinely used as autophagy inhibitors due to their suppressive effect on class III PI3Ks. Nevertheless, 3-MA with a prolonged period of treatment (up to 9 hours) was also found to promote autophagy even under nutrient-rich conditions. 3-MA also does not inhibit Beclin1-independent autophagy [205], however, it can still suppress starvation-induced autophagy [206]. In contrast, wortmannin suppresses autophagy regardless of the nutrient status [206]. The effect of 3-MA (but not wortmannin) is further complicated by differential temporal effects on class I and class III PI3Ks. 3-MA persistently blocks class I PI3Ks, whereas its impact on class III PI3Ks is only transient [206]. Furthermore, activation of autophagy was shown in gastric cancer cell line SGC7901 due to LY294002 treatment [207]. Another problem is that LY294002, and to a lesser extent wortmannin (but not 3-MA [208]), can inhibit proteosynthesis [209] and 3-MA can induce a decrease in cell viability not driven by the inhibition of the Akt/mTOR axis. The cytotoxicity induced by $3-\mathrm{MA}$ correlated with massive DNA damage monitored by $\gamma$-H2AX and was observed using the $10 \mathrm{mM}$ concentration, the usual concentration used to inhibit autophagy [210].

Off-target activities of PI3K inhibitors influence proteasome degradation, PI3K/Akt signalling pathways, endocytosis, lysosomal acidification, mitochondrial permeability transition, and glycogen metabolism [211, 212]. Moreover, it is known that PI3Ks also participate in the biogenesis of MVBs and their activity is required for the correct maturation of the ILVs [10, 129]. As would be expected, factors having some effect on the formation of MVBs also affect the secretion of exosomes. Consequently, conventional exosome production can be decreased by PI3K inhibitors [213-215]. Accordingly, wortmannin reduced the secretion of prostasomes from PC-3 cells [216]. On the other hand, inhibition of autophagy by wortmannin or CRISPR/ Cas9-mediated knockout of ATG5 greatly increased the release of exosome-associated prions [217]. In fibroblasts, wortmannin caused swollen endosome phenotype coupled with the failure of membrane recycling but not the inhibition of MVBs biogenesis [218]. Nevertheless, wortmannin can change the inner content of
ILVs [129]. The efficacy of wortmannin may be diminished by its covalent binding to free amino acids [219].

Inhibition of autophagy with 3-MA or wortmannin can have profound effects on cytokine secretion. In macrophages, toll-like receptor ligands initiate sequestration of pro-IL-1 $\beta$ into autophagosomes and activation of autophagy with rapamycin triggered the degradation of this sequestrated pro-IL-1 $\beta$ and blocked secretion of the mature cytokine. When treated with 3-MA or wortmannin, LPS-stimulated bone marrow-derived macrophages (iBMM) and dendritic cells (BMDC) secreted increased levels of IL-1 $\beta$. At the $10 \mathrm{mM}$ concentration, 3-MA induced IL- $1 \beta$ but inhibited IL- 6 secretion from BMDC and iBMM. In contrast, 3-MA and wortmannin markedly reduced IL-1 $\beta$ secretion induced by LPS + ATP in human neutrophils [220]. 3-MA, in combination with LPS, increased IL- $1 \alpha$ secretion by BMDC and IL-18 secretion by iBMM. 3-MA also activates the inflammasome through inhibition of type III PI3Ks [221]. Wortmannin was found to enhance IL-12 production in dendritic cells [222]. In contrast, LY294002 prevented IL-12 secretion in dendritic cells [209].

\section{VPS34 inhibitors}

VPS34 is involved not only in autophagy but also in the endosomal trafficking of receptors such as the epidermal growth factor receptor (EGFR) [129]. In addition to managing autophagy induction in complex I, VPS34 also has a role in the fusion of autophagosomes with lysosomes. Disruption of neuronal VPS34 function impairs autophagy, lysosomal degradation, as well as lipid metabolism, causing endolysosomal membrane damage. PI3P deficiency caused by a malfunction of VPS34 also promotes the secretion of unique exosomes enriched for undigested lysosomal substrates [223]. Considering the key role of VPS34 in autophagy, many compounds aim to target this kinase. The following section will present the characterization of VPS34 inhibitors Spautin-1, autophinib, SAR405, VPS34-IN1, and Cpd18.

Spautin-1 does not directly affect the catalytic activity of VPS34 but promotes the degradation of VPS34 complexes by inhibiting ubiquitin-specific peptidases USP10 and USP13. Under glucose-free conditions, Spautin-1 supports the ubiquitination of Beclin1 and triggers its degradation leading to destabilization and degradation of VPS34 complexes and inhibition of autophagy. VPS34 complexes also provide a molecular mechanism for class III PI3K to control the levels of p53. Therefore, levels of p53 are reduced in the tissues of BECN1+/mice [224]. Exosome production was found to be regulated by the p53 response as up-regulation of TSAP6 transcription by activated p53 can increase exosome release [225]. The destabilization of the PI3K complex 
that occurs upon suppressing Beclin1 (either via siRNAmediated knockdown or Spautin-1 treatment) reduced both exosome release and autophagy flux in chronic myeloid leukaemia cells [226]. Alternatively, Beclin1 may regulate autophagosome formation [227] and fusion of endosomes and autophagosomes leading to amphisome formation [228, 229]. In addition, it was also found that Beclin1 is needed for autophagosome fusion with lysosomes [230].

Autophinib is an ATP-competitive inhibitor of VPS34 decreasing the accumulation of the lipidated protein LC3 on the autophagosomal membrane. It inhibits autophagy induced by rapamycin or by amino acid starvation. The in vitro IC50 value for VPS34 is $19 \mathrm{nM}$ [231]. Since VPS34 has also a role in the fusion of autophagosomes with lysosomes, VPS34 inhibition causes autolysosomal dysfunction, a phenotype with large late endosomes and an enhanced release of atypical exosomes harbouring polyubiquitinated proteins [136-138].

SAR405 and VPS34-IN1 are highly potent and selective inhibitors of VPS34 not affecting the closely related class I and class II PI3Ks. SAR405 and VPS34-IN1 cause defects in autophagosome formation and endosomal trafficking [232]. SAR405 can inhibit autophagy induced by starvation and/or mTOR inhibition [233]. SAR405 prevents the catalytic activity of ATG14L and UVRAGcontaining VPS34 complexes, induces late endosome swelling, and affects late endosome-lysosome compartments [233]. Using SAR405 decreased the tumour growth and improved mouse survival in multiple tumour models by inducing tumour infiltration of NK, CD8+, and CD4+ T effector cells [96] and repressed viability of liver cancer stem cells [97]. Nevertheless, VPS34 function is critical in dendritic cells where it controls antigen cross-presenting. Consequently, VPS34 inhibition may lead to impaired T-cell-mediated immunity that may limit the use of SAR405 in anticancer therapy [99].

VPS34-IN1 selectively decreased PI3P levels, increased Beclin1 levels, but did not downregulate its other interacting partners from complex I, namely ATG14L, and VPS15 (in contrast to knockout of VPS34), ruling out indirect effects of destabilization of these proteins [223]. PI3P deficiency was shown to promote the secretion of unique exosomes enriched in undigested lysosomal substrates, including amyloid precursor protein C-terminal fragments, specific sphingolipids, and the phospholipid bis(monoacylglycero)phosphate. Secretion of these exosomes needs neutral sphingomyelinase 2 and sphingolipid synthesis. It was noted that proteins typically associated with exosomes such as Alix and CD63, or with ESCRT-dependent ILV sorting (TSG101 and Hrs) were minimally affected, suggesting that VPS34-IN1 likely affects composition rather than quantity of extracellular vesicles [223]. Neurons treated with VPS34-IN1 showed delayed degradation of EGFR following EGF stimulation. The remaining degradation was blocked by V-ATPase inhibitor bafilomycin A1, suggesting that VPS34 inhibition only partially impairs lysosomal function [223].

Cpd18 and 3-MA are structurally related compounds that differ only in a methyl piperidine group at the C6 of the adenine, but unlike 3-MA, Cpd18 does not inhibit class I PI3Ks. Cpd18 inhibits omegasome formation [234]. Nevertheless, the concentrations of Cpd18 that presented a greater attenuation of the autophagic flux are associated with cytotoxicity [210] and decreased ubiquitin/proteasome-dependent proteolysis in living cells [234].

\section{Late autophagy inhibitors}

Another way to inhibit autophagy is to target the later stages of the autophagy machinery, such as the fusion of autophagosomes with lysosomes and the degradation of autolysosome content by the lysosomal enzymes. Autophagosome-lysosome fusion involves the action of SNAREs. Autophagosome-lysosome fusion is orchestrated by the autophagosomal SNAREs STX17 and SNAP29, lysosomal R-SNARE VAMP8, HOPS tethering complex, small GTPase RAB7, and accessory proteins such as ATG14. Interestingly, the translocation of STX17 occurs only on complete autophagosomes and not on partially formed autophagosomes. EACC blocks autophagosome-lysosome fusion by preventing STX17 and SNAP29 loading onto autophagosomes without impeding the completion of autophagosomes. It also causes an accumulation of LC3-II and reduces the interaction of STX17 with the HOPS subunit VPS33A and the lysosomal VAMP8. On the other hand, autophagy induction, the number of autophagosome biogenesis sites, expansion of the phagophore, lysosomal $\mathrm{pH}$, localization of lysosomal SNAREs or RABs, and cargo recognition remain unaltered in the presence of EACC [235] although STX17 was also shown to be involved in autophagy initiation [46]. Interestingly, STX17 depletion increased the production of exosomes in A549 cells [236] and effectively blocks the formation of axonal amphisomes after 3 hours starvation in dorsal root ganglion neurons [237].

Chloroquine (CQ) and its derivatives (such as 3-hydroxychloroquine HCQ or Lys05) inhibit the maturation of autolysosomes and block late steps of autophagy. In contradiction with previous studies [238], some more recent studies indicate that CQ does not substantially decrease lysosomal acidity, and the lysosomes retain their capacity to degrade delivered material [239]. Although CQ may induce a temporal elevation of lysosomal $\mathrm{pH}$, this elevation may be only transient and can be followed by reacidification of the lysosomes [240]. 
The kinetics of this transient phase may differ between cell types [239]. The greater part of the confusion about CQ effects on lysosomal $\mathrm{pH}$ might be attributed to how it was measured because LysoTracker Red dye, often used to estimate lysosomal $\mathrm{pH}$, is not a $\mathrm{pH}$ sensor and the intensity of its fluorescence signal does not correlate with the lysosomal pH [239]. Nevertheless, CQ behaves as a weak base and accumulates in the lysosomes causing lysosomal stress. Lysosomal stress may cause the release of EVs to eliminate cellular waste [223]. Accordingly, the production of exosomes was increased due to CQ treatment [236]. CQ treatment led to marked lysosomal swelling and recruitment of Galectin-3 to sites of membrane damage [241]. In response to lysosomal damage, IL-1 $\beta$ can be recognized by secretory autophagy cargo receptor TRIM16 [176]. Strikingly, glucose starvation or hexokinase inhibition by 2-deoxyglucose prevented CQ from inducing lysosomal damage and subsequent cell death [241]. Accordingly, IL-1 $\beta$ release correlates with the degree of lysosome damage [242] and glucose is required for LPS-induced IL-1 $\beta$ production by monocytes [243]. Furthermore, autophagy inhibition with $C Q$ also induced the secretion of pro-inflammatory cytokines MIF (Macrophage migration inhibitory factor) and IL-6 in triplenegative breast cancer cells [244].

Although CQ and HCQ are indisputably impairing the autophagic flux, their use entails multiple side effects including the disorganization of the Golgi and endo-lysosomal networks, dysregulation of STX17 and SNAP29 targeting, and even a temporary induction of autophagic sequestration activity (probably by inhibiting mTORC1 in a Rag-dependent manner [245]) and a drop in ATP content [227, 239]. Furthermore, during starvation or CQ-induced lysosomal stress, TFEB and TFE3 rapidly translocate to the nucleus to initiate lysosomal biogenesis [240]. CQ was also shown to increase amphisome and IFN- $\alpha$ production in human plasmacytoid dendritic cells stimulated by the Herpes simplex virus. On the other hand, when Beclin1 was knocked down, virus-induced IFN- $\alpha$ production was significantly suppressed [246].

Bafilomycin A1 (BAFA1) is a V-ATPase inhibitor that blocks the autophagic flux by inhibiting autophagosomelysosome fusion (possibly by inhibiting the ATP2A/ SERCA pump [247]) and autolysosomal and/or lysosomal acidification. It also reduces the delivery of internalized molecules from MVBs to lysosomes [248]. On the other hand, trafficking through early and late endosomes continues upon BAFA1 treatment [249]. BAFA1-treated cells display phenotypes associated with an inhibition of the degradation capacity of lysosomes such as the presence of intact cytoplasm in the lysosomal lumen and a loss of acidity [239]. BAFA1 inhibits lysosomal degradation and thereby negatively affects the amino acid efflux from the lysosomes, possibly impairing mTOR signalling which is dependent on this organelle (mTOR localizes to lysosomes and its activation depends on amino acids inside the lysosomal lumen) [245]. mTOR inhibition strongly decreased exosomal prion release [217]. In contrast, starvation stimulated exosome release through a RAB27a-dependent mechanism but did not significantly alter exosomal cargo content [250]. BAFA1 also triggers BAX- and/or BAK-dependent cytotoxicity and caspase activation [210] and has indirect effects on Golgi trafficking [251]. According to some indications, BAFA1 can also inhibit signals from the lysosomal P5-type ATPase ATP13A2 (also known as PARK9) [252]. ATP13A2 has been found to regulate both autophagic degradation and exosomal release [253]. Elevated levels of ATP13A2 enhance the externalization of $\alpha$-synuclein through amphisomal structures, which is proposed to be accomplished through ATP13A2-mediated modulation of intraluminal zinc ion levels in MVBs [254, 255]. On the other hand, the exosomal release was enhanced due to BAFA1 treatment $[153,236]$. Inhibition of autophagy with BAFA1 markedly reduced IL- $1 \beta$ secretion induced by LPS+ATP in human neutrophils [220].

The principal enzymatic activity of PIKfyve (phosphoinositide kinase, FYVE-type zinc finger containing) is to phosphorylate PI3P to PI (3,5)P2. PIKfyve inhibitors, such as YM201636, vacuolin-1, and apilimod mesylate, disrupt lysosome turnover, the heterotypic fusion of lysosomes with autophagosomes and/or MVBs, and the formation of autolysosomes resulting in autophagy inhibition [256]. Acute inhibition of PIKfyve also blocks protein sorting and their turnover in late endosomes. Both $\operatorname{PI}(3,5)$ P2-deficient cells and cells that lack TRPML1 exhibited enlarged endolysosomes and trafficking defects in the late endocytic pathway [257]. Furthermore, PI(3,5)P2 is required for lysosomal acidification by the V-ATPase [258]. Consequently, PIKfyve inhibition enhances exosome release and triggers secretory autophagy in PC-3 cells (probably to relieve stress caused by disruption of recycling pathways). These exosomes bear the typical exosomal markers (TSG101, Alix) and a subset of ATGs [259].

\section{Conclusion}

Autophagy and MVBs-related secretory pathways are interconnected at many levels. These pathways, collectively with the ubiquitin-proteasome system, orchestrate the dynamics of intracellular waste removal, where each pathway may complement the deficiencies of the other. In other words, exosome secretion can reduce stress when degradative and recycling pathways are disrupted. On the other hand, unwanted MVBs with damaged material may be directed to lysosomes. Furthermore, some parts 


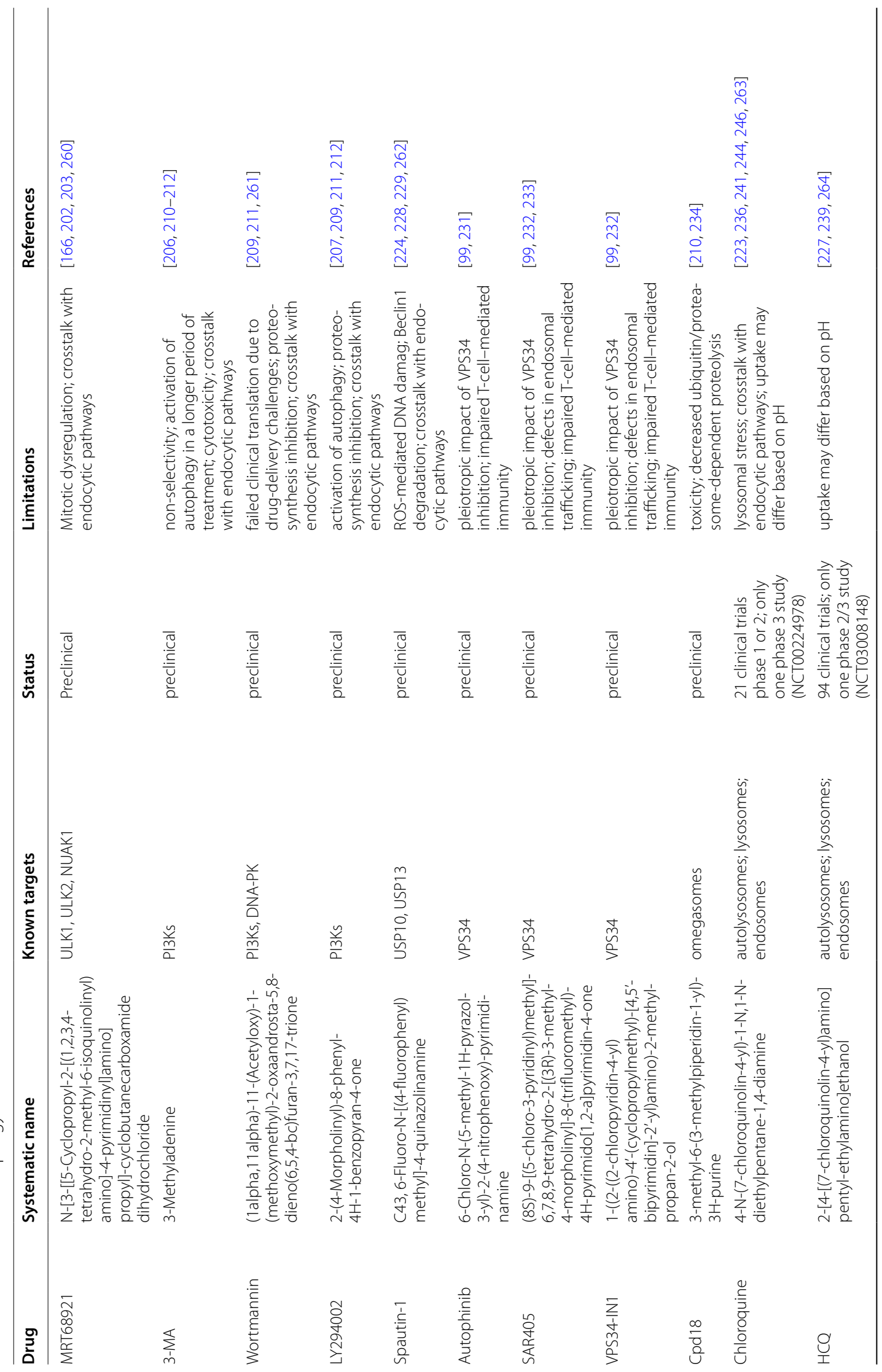




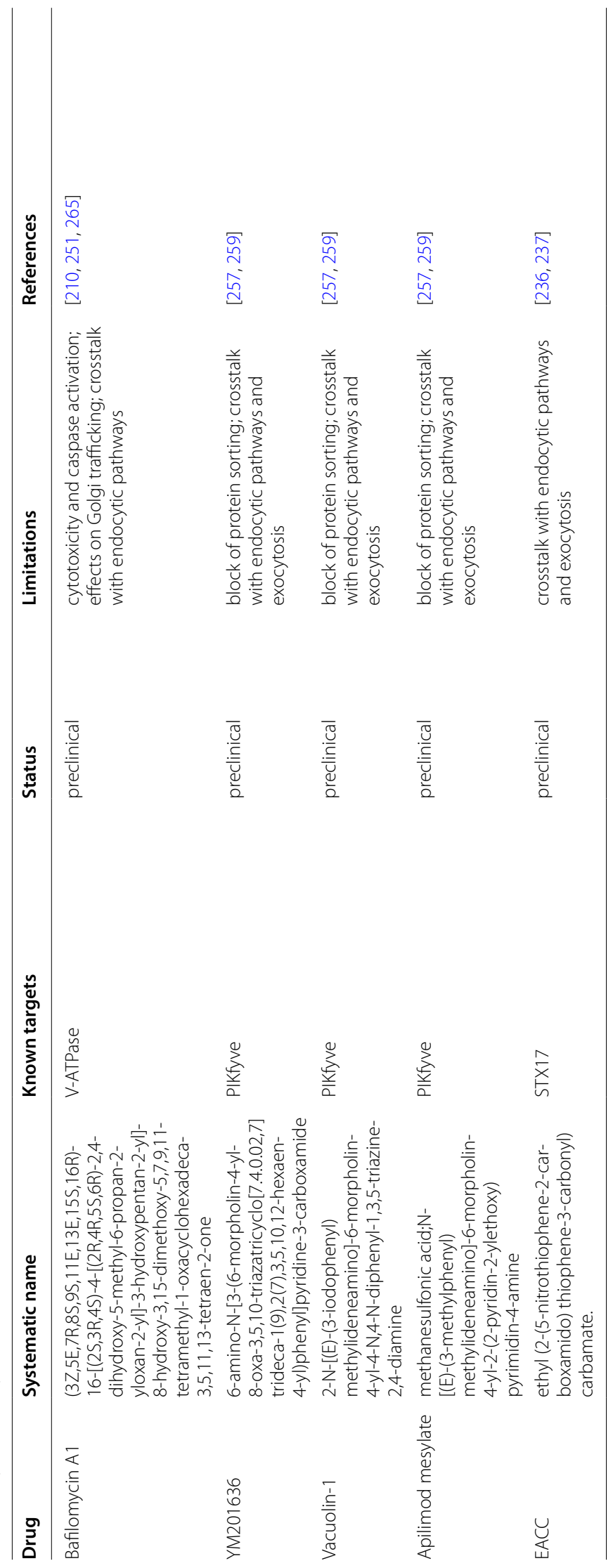


of functional autophagy machinery are important for the genesis of endosomes and amphisomes. Consequently, autophagy inhibition can both promote and/or decrease EVs release. The resulting effect is largely contextdependent and could be significantly affected by different kinds of autophagy modulators. Moreover, modulation of autophagy significantly impacts not only EVs quantity but probably also their content. Late and early autophagy inhibitors can have a profoundly different effect on secretion. For example, Spautin-1 and CQ are both autophagy inhibitors but have nearly opposite effects on EVs release. Many studies suggest that cancer cells release higher amounts of EVs compared to non-malignant cells, which makes the effect of autophagy inhibitors on EVs secretion highly important and attractive for anticancer therapy. In future studies, it should be carefully assessed how exactly autophagy could be targeted (late versus early autophagy inhibitors) to maximize patient benefit and improve cancer therapy. For safe and successful clinical use of autophagy inhibitors, we need to carefully explore the molecular mechanisms underlying the effects of autophagy on tumour progression and possibly discover all pathways affected by particular autophagy inhibitors (see Table 2).

\footnotetext{
Abbreviations

3-MA: 3-methyladenine; AKT: Rac-alpha serine/threonine-protein kinase; Alix: Apoptosis-linked gene 2-interacting protein X; AMBRA1: Autophagy and Beclin 1 regulator 1; AMPK: AMP-activated protein kinase; ANXA2: Annexin 2; Arf6: ADP ribosylation factor 6; ATG: Autophagy-related genes; ATP: Adenosine triphosphate; ATP13A2: ATPase cation transporting 13A2; Beclin1: Coiled-coil, moesin-like BCL2 interacting protein; Bif-1: Bax-interacting factor 1; BMDC: Bone marrow-derived dendritic cells; CaMKKB: Calcium/calmodulin-dependent protein kinase kinase $\beta$; CCL: C-C motif chemokine ligand; COPII: Coat protein complex Il; CQ: Chloroquine; CSF3/G-CSF: Granulocyte colony-stimulating factor; C-Src: Proto-Oncogene Tyrosine-Protein Kinase Src; CXCL: C-X-C motif chemokine ligand ${ }_{i}$ DEPTOR: DEP domain-containing mTOR interacting protein; DFCP1: Double FYVE-containing protein 1; EGFR: Epidermal growth factor receptor; EMT: Epithelial to mesenchymal transition; ER: Endoplasmic reticulum; ESCRT: Endosomal sorting complexes required for transport; EVs: Extracellular vesicles; FIP200: FAK family-interacting protein of 200 kDa; G3BP: Ras GTPase-activating protein SH3-domain-binding protein; GDP: Guanosine diphosphate; GTP: Guanosine triphosphate; GTPase: Guanosine triphosphatase; HCQ: Hydroxychloroquine; HOPS: Homotypic fusion, and protein sorting complex; CHMP: Charged multivesicular body protein; iBMM: Bone marrow-derived macrophages; IFN-a: Interferon alpha; IKKB: IKB kinase; IL: Interleukin; ILVs: Intraluminal vesicles; ISG15: Interferon (IFN)-a/ $\beta$-induced ubiquitin-like protein; LAMP2: Lysosome-associated membrane protein 2; LC3: Microtubule-associated protein 1A/1B-light chain 3; LKB1: Liver kinase B1; LPS: Bacterial lipopolysaccharides; MGRN1: Mahogunin ring finger 1; MHC: Major histocompatibility complex (MHC-II); MIF: Macrophage migration inhibitory factor; $\mathrm{mLST}$ 8: Mammalian lethal with Sec13 protein 8, alias GBL; MMP: Matrix metalloproteinase; mTOR: Mammalian target of rapamycin; mTORC: Mammalian target of rapamycin complex; MVBs: Multivesicular bodies; NF-kB: Nuclear factor-kappa B; NUAK1: NUAK family SNF1-like kinase 1; p115: Vesicular transport factor p115; PARK7: Parkinsonism-associated deglycase 7; PI $(3,4,5)$ P3: Phosphatidylinositol (3,4,5)-trisphosphate; PI3K: Phosphoinositide 3-kinase; PI3P: Phosphatidylinositide-3-phosphate; PIKfyve: Phosphoinositide kinase, FYVE-type zinc finger containing; PKA: Protein kinase A; PLD2: Phospholipase D2; PRAS40: Proline-rich Akt substrate of 40 kDa; Rab: Ras-associated GTP-binding protein; Rag: Ras-related GTP-binding protein; RAPTOR:
}

Regulatory-associated protein of mTOR also known as KIAA1303; Rheb: Ras homolog enriched in brain; SEC22b: SEC22 vesicle-trafficking protein homolog B; shRNA: Small hairpin RNA; siRNA: Small interfering RNA; SNAP29: Synaptosome-associated protein 29; SNARE: Soluble N-ethylmaleimidesensitive fusion attachment protein receptors; STAT3: Signal transducer and activator of transcription 3; STX17: Syntaxin-17; TBC-2: TBC domain-containing protein 2; TFE3: Transcription factor binding to IGHM Enhancer 3; TFEB:Transcription factor EB; TME: Tumour microenvironment; TREM1: Triggering receptor expressed on myeloid cells 1;TRPML 1: Transient receptor potential channel mucolipin 1;TSC: Tuberous sclerosis complex; TSG101: Tumuor susceptibility 101; ULK: Unc51-like autophagy-activating kinase; UVB: Ultraviolet light B; UVRAG: UV resistance-associated gene; VAMP: Vesicle-associated membrane protein; V-ATPase: V1V0-vacuolar-type ATPase; VPS: Vacuolar protein sorting; WIPI: WD repeat domain phosphoinositide-interacting protein; $\gamma-\mathrm{H} 2 \mathrm{AX}$ : Phosphorylation of the Ser-139 of the histone variant H2AX.

\section{Acknowledgements}

Not applicable

\section{Authors' contributions}

MR wrote the manuscript, JB conceived the structure and revised the manuscript, MM revised the manuscript. All authors read and approved the final manuscript.

\section{Funding}

This work was supported by the Ministry of Health of the Czech Republic (NU20J-08-00018), by Grant Agency of the Czech Republic (GACR 21-06873S), by funds from Specific University Research Grant, as provided by the Ministry of Education, Youth and Sports of the Czech Republic in the year 2021 (MUNI/A/1698/2020 and MUNI/A/1246/2020), by the "Center for Tumor Ecology - Research of the Cancer Microenvironment Supporting Cancer Growth and Spread" (reg. no. CZ.02.1.01/0.0/0.0/16_019/0000785) supported by the Operational Program Research, Development and Education and by the Ministry of Education, Youth and Sports of the Czech Republic, project Advanced Functional Nanorobots reg. No. CZ.02.1.01/0.0/0.0/15_003/0000444.

\section{Availability of data and materials}

Not applicable

\section{Declarations}

Ethics approval and consent to participate

Not applicable

\section{Consent for publication}

Not applicable

\section{Competing interests}

The authors declare that they have no competing interests.

\section{Author details}

${ }^{1}$ Department of Physiology, Faculty of Medicine, Masaryk University, Kamenice 5, CZ-625 00 Brno, Czech Republic. ${ }^{2}$ Department of Chemistry and Biochemistry, Mendel University in Brno, Zemedelska 1, CZ-613 00 Brno, Czech Republic. ${ }^{3}$ Department of Pathological Physiology, Faculty of Medicine, Masaryk University, Kamenice 5, CZ-625 00 Brno, Czech Republic. ${ }^{4}$ BIOCEV, First Faculty of Medicine, Charles University, Prumyslova 595, CZ-252 50 Vestec, Czech Republic. ${ }^{5}$ Center for Advanced Functional Nanorobots, Department of Inorganic Chemistry, Faculty of Chemical Technology, University of Chemistry and Technology in Prague, Technická 5, CZ-166 28 Prague, Czech Republic.

Received: 1 June 2021 Accepted: 11 September 2021

Published online: 27 October 2021

\section{References}

1. Mizushima N. Physiological functions of autophagy. Curr Top Microbiol Immunol. 2009;335:71-84. 
2. Clarke AJ, Simon AK. Autophagy in the renewal, differentiation and homeostasis of immune cells. Nat Rev Immunol. 2019;19(3):170-83.

3. Deretic V, Jiang S, Dupont N. Autophagy intersections with conventional and unconventional secretion in tissue development, remodeling and inflammation. Trends Cell Biol. 2012;22(8):397-406.

4. Salimi L, et al. Synergies in exosomes and autophagy pathways for cellular homeostasis and metastasis of tumor cells. Cell Biosci. 2020;10(1):64.

5. Kaushik S, Cuervo AM. The coming of age of chaperone-mediated autophagy. Nat Rev Mol Cell Biol. 2018;19(6):365-81.

6. Jovic M, et al. The early endosome: a busy sorting station for proteins at the crossroads. Histol Histopathol. 2010;25(1):99-112.

7. Barysch SV, et al. Sorting in early endosomes reveals connections to docking- and fusion-associated factors. Proc Natl Acad Sci. 2009;106(24):9697-702.

8. Hessvik NP, Llorente A. Current knowledge on exosome biogenesis and release. Cell Mol Life Sci. 2018;75(2):193-208.

9. Murrow L, Malhotra R, Debnath J. ATG12-ATG3 interacts with Alix to promote basal autophagic flux and late endosome function. Nat Cell Biol. 2015;17(3):300-10

10. Fader $\mathrm{CM}$, et al. Induction of autophagy promotes fusion of multivesicular bodies with autophagic vacuoles in $\mathrm{K} 562$ cells. Traffic. 2008;9(2):230-50.

11. Abels ER, Breakefield XO. Introduction to extracellular vesicles: biogenesis, RNA cargo selection, content, release, and uptake. Cell Mol Neurobiol. 2016;36(3):301-12

12. Ao X, Zou L, Wu Y. Regulation of autophagy by the Rab GTPase network. Cell Death Differ. 2014;21(3):348-58.

13. Fengsrud $M$, et al. Ultrastructural characterization of the delimiting membranes of isolated autophagosomes and amphisomes by freeze-fracture electron microscopy. Eur J Cell Biol. 2000;79(12):871-82

14. Lucocq J, Walker D. Evidence for fusion between multilamellar endosomes and autophagosomes in HeLa cells. Eur J Cell Biol. 1997;72(4):307-13.

15. Klionsky DJ, Eskelinen E-L, Deretic V. Autophagosomes, phagosomes, autolysosomes, phagolysosomes, autophagolysosomes... wait, I'm confused. Autophagy. 2014;10(4):549-51.

16. Klionsky DJ, et al. Guidelines for the use and interpretation of assays for monitoring autophagy (4th edition)(1). Autophagy. 2021;17(1):1-382.

17. Saxton RA, Sabatini DM. mTOR signaling in growth, metabolism, and disease. Cell. 2017;168(6):960-76.

18. Jewell JL, et al. GPCR signaling inhibits mTORC1 via PKA phosphorylation of Raptor. Elife. 2019;8.

19. Gwinn DM, et al. AMPK phosphorylation of raptor mediates a metabolic checkpoint. Mol Cell. 2008:30(2):214-26.

20. Prentzell MT, et al. G3BPs tether the TSC complex to lysosomes and suppress mTORC1 signaling. Cell. 2021.

21. González A, Hall MN. Nutrient sensing and TOR signaling in yeast and mammals. EMBO J. 2017:36(4):397-408.

22. Sancak Y, et al. Ragulator-Rag complex targets mTORC1 to the lysosomal surface and is necessary for its activation by amino acids. Cell. 2010;141(2):290-303.

23. Zoncu $\mathrm{R}$, et al. mTORC1 senses lysosomal amino acids through an inside-out mechanism that requires the vacuolar $\mathrm{H}+$-ATPase. Science. 2011;334:678-83.

24. Mutvei AP, et al. Rap1-GTPases control mTORC1 activity by coordinating lysosome organization with amino acid availability. Nat Commun. 2020:11(1):1416.

25. Settembre C, et al. TFEB links autophagy to lysosomal biogenesis. Science. 2011;332(6036):1429-33.

26. Kundu ST, et al. TMEM106B drives lung cancer metastasis by inducing TFEB-dependent lysosome synthesis and secretion of cathepsins. Nat Commun. 2018;9(1):2731.

27. Karanasios $\mathrm{E}$, et al. Autophagy initiation by ULK complex assembly on ER tubulovesicular regions marked by ATG9 vesicles. Nat Commun. 2016:7:12420

28. Karanasios E, et al. Dynamic association of the ULK1 complex with omegasomes during autophagy induction. J Cell Sci. 2013:126(22):5224-38
29. Shima T, Kirisako H, Nakatogawa H. COPII vesicles contribute to autophagosomal membranes. J Cell Biol. 2019;218(5):1503-10.

30. Imai K, et al. Atg9A trafficking through the recycling endosomes is required for autophagosome formation. J Cell Sci. 2016;129(20):3781-91.

31. Puri C, et al. ATG16L1 meets ATG9 in recycling endosomes: additional roles for the plasma membrane and endocytosis in autophagosome biogenesis. Autophagy. 2014;10(1):182-4.

32. Lamb CA, Yoshimori T, Tooze SA. The autophagosome: origins unknown biogenesis complex. Nat Rev Mol Cell Biol. 2013;14(12):759-74.

33. Boukhalfa A, et al. PI3KC2a-dependent and VPS34-independent generation of PI3P controls primary cilium-mediated autophagy in response to shear stress. Nat Commun. 2020;11(1):294.

34. Chowdhury S, et al. Insights into autophagosome biogenesis from structural and biochemical analyses of the ATG2A-WIPI4 complex. Proc Natl Acad Sci. 2018;115(42):E9792.

35. Maeda S, Otomo C, Otomo T. The autophagic membrane tether ATG2A transfers lipids between membranes. Elife. 2019;8.

36. Dancourt J, Melia TJ. Lipidation of the autophagy proteins LC3 and GABARAP is a membrane-curvature dependent process. Autophagy. 2014;10(8):1470-1.

37. Tanida I, Ueno T, Kominami E. LC3 conjugation system in mammalian autophagy. Int J Biochem Cell Biol. 2004;36(12):2503-18.

38. Kharaziha P, Panaretakis T. Dynamics of Atg5-Atg12-Atg16L1 Aggregation and Deaggregation. Methods Enzymol. 2017;587:247-55.

39. Agrotis A, et al. Redundancy of human ATG4 protease isoforms in autophagy and LC3/GABARAP processing revealed in cells. Autophagy. 2019;15(6):976-97.

40. Li M, et al. Kinetics comparisons of mammalian Atg4 homologues indicate selective preferences toward diverse Atg8 substrates. J Biol Chem. 2011:286(9):7327-38.

41. Takahashi Y, et al. An autophagy assay reveals the ESCRT-III component CHMP2A as a regulator of phagophore closure. Nat Commun. 2018;9(1):2855

42. Weidberg H, et al. LC3 and GATE-16/GABARAP subfamilies are both essential yet act differently in autophagosome biogenesis. EMBO J. 2010;29(11):1792-802.

43. Grunwald DS, et al. GABARAPs and LC3s have opposite roles in regulating ULK1 for autophagy induction. Autophagy. 2020;16(4):600-14

44. Yamamoto M, Suzuki SO, Himeno M. The effects of dynein inhibition on the autophagic pathway in glioma cells. Neuropathology. 2010;30(1):1-6.

45. Tong J, Yan X, Yu L. The late stage of autophagy: cellular events and molecular regulation. Protein Cell. 2010;1 (10):907-15.

46. Kumar S, et al. Phosphorylation of Syntaxin 17 by TBK1 controls autophagy initiation. Dev Cell. 2019;49(1):130-44 e6.

47. Wang $\mathrm{C}$, et al. Phosphorylation of ULK1 affects autophagosome fusion and links chaperone-mediated autophagy to macroautophagy. Nat Commun. 2018;9(1):3492.

48. Viret C, Faure M. Regulation of syntaxin 17 during autophagosome maturation. Trends Cell Biol. 2019;29(1):1-3.

49. Choi YJ, et al. Inhibitory effect of mTOR activator MHY1485 on autophagy: suppression of lysosomal fusion. PLoS One. 2012;7(8)::43418.

50. Itakura E, et al. Beclin 1 forms two distinct phosphatidylinositol 3-kinase complexes with mammalian Atg14 and UVRAG. Mol Biol Cell. 2008;19(12):5360-72.

51. Liang C, et al. Beclin1-binding UVRAG targets the class C Vps complex to coordinate autophagosome maturation and endocytic trafficking. Nat Cell Biol. 2008:10(7):776-87.

52. Takahashi Y, et al. Bif-1 interacts with Beclin 1 through UVRAG and regulates autophagy and tumorigenesis. Nat Cell Biol. 2007:9(10):1142-51.

53. Keulers TG, Schaaf MBE, Rouschop KMA. Autophagy-dependent secretion: contribution to tumor progression. Front Oncol. 2016;6(251).

54. Liang $\mathrm{XH}$, et al. Induction of autophagy and inhibition of tumorigenesis by beclin 1. Nature. 1999;402(6762):672-6.

55. Yue $Z$, et al. Beclin 1, an autophagy gene essential for early embryonic development, is a haploinsufficient tumor suppressor. Proc Natl Acad Sci U S A. 2003;100(25):15077-82. 
56. Kang MR, et al. Frameshift mutations of autophagy-related genes ATG2B, ATG5, ATG9B and ATG12 in gastric and colorectal cancers with microsatellite instability. J Pathol. 2009;217(5):702-6.

57. Wible DJ, et al. ATG5 cancer mutations and alternative mRNA splicing reveal a conjugation switch that regulates ATG12-ATG5-ATG16L1 complex assembly and autophagy. Cell Discov. 2019;5:42.

58. Ueno T, et al. Loss of Pten, a tumor suppressor, causes the strong inhibition of autophagy without affecting LC3 lipidation. Autophagy. 2008:4(5):692-700.

59. Hou W, et al. Mutation analysis of key genes in RAS/RAF and PI3K/PTEN pathways in Chinese patients with hepatocellular carcinoma. Oncol Lett. 2014;8(3):1249-54.

60. Chen $X$, et al. DEPTOR is an in vivo tumor suppressor that inhibits prostate tumorigenesis via the inactivation of $\mathrm{mTORC} 1 / 2$ signals. Oncogene. 2020;39(7):1557-71.

61. Johnson CE, et al. Loss of tuberous sclerosis complex 2 sensitizes tumors to nelfinavir-bortezomib therapy to intensify endoplasmic reticulum stress-induced cell death. Oncogene. 2018;37(45):5913-25.

62. Chang WH, Lai AG. An integrative pan-cancer investigation reveals common genetic and transcriptional alterations of AMPK pathway genes as important predictors of clinical outcomes across major cancer types. BMC Cancer. 2020;20(1):773.

63. Zijian, Z., et al., Research square, 2021.

64. Claude-Taupin A, et al. ATG9A is overexpressed in triple negative breast cancer and its in vitro extinction leads to the inhibition of pro-cancer phenotypes. Cells. 2018;7(12):248.

65. Caron A, et al. DEPTOR at the nexus of cancer, metabolism, and immunity. Physiol Rev. 2018;98(3):1765-803.

66. Hu B, et al. Downregulation of DEPTOR inhibits the proliferation, migration, and survival of osteosarcoma through PI3K/Akt/mTOR pathway. Onco Targets Ther. 2017;10:4379-91.

67. Chen R, Yang Q, Lee J-D. BMK1 kinase suppresses epithelial-mesenchymal transition through the Akt/GSK3 3 signaling pathway. Cancer Res. 2012;72(6):1579-87.

68. Parvani JG, et al. Deptor enhances triple-negative breast cancer metastasis and chemoresistance through coupling to survivin expression. Neoplasia. 2015;17(3):317-28.

69. Pei $L$, et al. Overexpression of DEP domain containing mTOR-interacting protein correlates with poor prognosis in differentiated thyroid carcinoma. Mol Med Rep. 2011;4(5):817-23.

70. Okamoto T, et al. FIP200 suppresses immune checkpoint therapy responses in breast cancers by limiting AZI2/TBK1/IRF signaling independent of its canonical autophagy function. Cancer Res. 2020;80(17):3580-92.

71. Wang D, et al. Downregulation of FIP200 induces apoptosis of glioblastoma cells and microvascular endothelial cells by enhancing Pyk2 activity. PLoS One. 2011;6(5):e19629.

72. Yu X-N, et al. Enhanced $m L S T 8$ expression correlates with tumor progression in hepatocellular carcinoma. Ann Surg Oncol. 2020;27(5):1546-57.

73. Chen $\mathrm{S}$, et al. Inhibition of PI3K/Akt/mTOR signaling in PI3KR2overexpressing colon cancer stem cells reduces tumor growth due to apoptosis. Oncotarget. 2017:8(31):50476-88.

74. Mossmann D, Park S, Hall MN. mTOR signalling and cellular metabolism are mutual determinants in cancer. Nat Rev Cancer. 2018;18(12):744-57.

75. Zou Z, et al. mTOR signaling pathway and mTOR inhibitors in cancer: progress and challenges. Cell Biosci. 2020;10(1):31.

76. Qi Z, et al. PRAS40 hyperexpression promotes hepatocarcinogenesis. EBioMedicine. 2020;51.

77. Zhu G, et al. PRAS40 promotes NF-KB transcriptional activity through association with p65. Oncogenesis. 2017;6(9):e381.

78. Nussinov R, et al. The mystery of Rap1 suppression of oncogenic ras. Trends Cancer. 2020;6(5):369-79.

79. Zhang $Y-L$, et al. Roles of Rap1 signaling in tumor cell migration and invasion. Cancer Biol Med. 2017;14(1):90-9.

80. Earwaker $\mathrm{P}$, et al. RAPTOR up-regulation contributes to resistance of renal cancer cells to PI3K-mTOR inhibition. PLoS One. 2018;13(2):e0191890.

81. Wang T, et al. RAPTOR promotes colorectal cancer proliferation by inducing $\mathrm{mTORC} 1$ and upregulating ribosome assembly factor URB1. Cancer Med. 2020:9(4):1529-43.
82. Kondo $S$, et al. Raptor and rictor expression in patients with human papillomavirus-related oropharyngeal squamous cell carcinoma. BMC Cancer. 2021;21(1):87.

83. Kauffman EC, et al. Molecular genetics and cellular features of TFE 3 and TFEB fusion kidney cancers. Nat Rev Urol. 2014;11 (8):465-75.

84. Kim JH, et al. TFEB supports pancreatic cancer growth through the transcriptional regulation of glutaminase. Cancers. 2021:13(3):483.

85. Li Y, et al. TFEB is a master regulator of tumor-associated macrophages in breast cancer. J ImmunoTher Cancer. 2020;8(1):e000543.

86. Medina DL, et al. Transcriptional activation of lysosomal exocytosis promotes cellular clearance. Dev Cell. 2011;21(3):421-30.

87. Astanina E, Bussolino F, Doronzo G. Multifaceted activities of transcription factor EB in cancer onset and progression. Mol Oncol. 2021;15(2):327-46.

88. Astrinidis A, et al. Tuberin, the tuberous sclerosis complex 2 tumor suppressor gene product, regulates Rho activation, cell adhesion and migration. Oncogene. 2002;21(55):8470-6.

89. Deng J, et al. ULK1 inhibition overcomes compromised antigen presentation and restores antitumor immunity in LKB1-mutant lung cancer. Nat Can. 2021;2(5):503-14.

90. Kumar M, Papaleo E. A pan-cancer assessment of alterations of the kinase domain of ULK1, an upstream regulator of autophagy. Sci Rep. 2020;10(1):14874

91. TAN L, TAN Y, LIU D. Functions of ULK1 in autophagy and nonautophagy pathways and its implications in human physiology and disease. Biocell. 2020:44(4):535-43.

92. Li Z, et al. ULK1-ATG13 and their mitotic phospho-regulation by CDK1 connect autophagy to cell cycle. PLoS Biol. 2020;18(6):e3000288.

93. De Luca M, Romano R, Bucci C. Role of the V1G1 subunit of V-ATPase in breast cancer cell migration. Sci Rep. 2021;11(1):4615.

94. Huang $L$, et al. ABCG2N-ATPase was associated with the drug resistance and tumor metastasis of esophageal squamous cancer cells. Diagn Pathol. 2012;7:180.

95. Lu Q et al. The expression of V-ATPase is associated with drug resistance and pathology of non-small-cell lung cancer. Diagn Pathol. 2013;8:145.

96. Noman MZ, et al. Inhibition of Vps34 reprograms cold into hot inflamed tumors and improves anti-PD-1/PD-L1 immunotherapy. Sci Adv. 2020;6(18):eaax7881.

97. Liu F, et al. PIK3C3 regulates the expansion of liver CSCs and PIK3C3 inhibition counteracts liver cancer stem cell activity induced by PI3K inhibitor. Cell Death Dis. 2020;11(6):427.

98. Jiang $\mathrm{X}$, et al. VPS34 stimulation of p62 phosphorylation for cancer progression. Oncogene. 2017;36.

99. Parekh W, et al. Autophagy-related protein Vps34 controls the homeostasis and function of antigen cross-presenting CD8a $<$ sup $\rangle+</$ sup $\rangle$ dendritic cells. Proc Natl Acad Sci. 2017:114(31):E6371-80.

100. Liang T-T, et al. Systemic expression analysis reveals prognostic significance of WIPI3 in hepatocellular carcinoma. Front Genet. 2020;1 1(847).

101. Kean MJ, et al. VAMP3, syntaxin-13 and SNAP23 are involved in secretion of matrix metalloproteinases, degradation of the extracellular matrix and cell invasion. J Cell Sci. 2009;122(Pt 22):4089-98.

102. Sneeggen M, Schink KO, Stenmark H. Tumor suppression by control of matrix metalloproteinase recycling. Mol Cell Oncol. 2019;6(6):e1646606.

103. Miao Y, et al. GABARAP is overexpressed in colorectal carcinoma and correlates with shortened patient survival. Hepatogastroenterology. 2010;57(98):257-61.

104. Liu Y, et al. GABARAP suppresses EMT and breast cancer progression via the AKT/mTOR signaling pathway. Aging. 2021;13(4):5858-74.

105. Othman EQ, et al. Immunohistochemical expression of MAP1LC3A and MAP1LC3B protein in breast carcinoma tissues. J Clin Lab Anal. 2009;23(4):249-58.

106. Kang HM, et al. Ubiquitination of MAP1LC3B by pVHL is associated with autophagy and cell death in renal cell carcinoma. Cell Death Dis. 2019;10(4):279.

107. Li X, He S, Ma B. Autophagy and autophagy-related proteins in cancer Mol Cancer. 2020;19(1):12

108. Sadler JBA, et al. A cancer-associated polymorphism in ESCRT-III disrupts the abscission checkpoint and promotes genome instability. Proc Natl Acad Sci U S A. 2018;115(38):E8900-8.

109. Song Y, Quach C, Liang C. UVRAG in autophagy, inflammation, and cancer. Autophagy. 2020;16(2):387-8. 
110. Ahn CH, et al. Expression of beclin-1, an autophagy-related protein, in gastric and colorectal cancers. APMIS. 2007;115(12):1344-9.

111. Hao M, Yeo SK, Guan J-L. Autophagy inhibition perturbs ERBB2 trafficking and abolishes tumorigenesis in ERBB2-driven breast cancer. Autophagy. 2021;17(4):1059-60

112. Cararo-Lopes E, et al. Autophagy buffers Ras-induced genotoxic stress enabling malignant transformation in keratinocytes primed by human papillomavirus. Cell Death Dis. 2021;12(2):194.

113. Jiang G-M, et al. The relationship between autophagy and the immune system and its applications for tumor immunotherapy. Mol Cancer. 2019;18(1):17.

114. Fung C, et al. Induction of autophagy during extracellular matrix detachment promotes cell survival. Mol Biol Cell. 2008;19(3):797-806.

115. Nazio F, et al. Autophagy and cancer stem cells: molecular mechanisms and therapeutic applications. Cell Death Differ. 2019;26(4):690-702.

116. Pérez-Hernández $\mathrm{M}$, et al. Targeting autophagy for cancer treatment and tumor chemosensitization. Cancers. 2019;11(10):1599.

117. Maes $\mathrm{H}$, et al. Autophagy: shaping the tumor microenvironment and therapeutic response. Trends Mol Med. 2013;19(7):428-46.

118. Sharifi MN, et al. Autophagy promotes focal adhesion disassembly and cell motility of metastatic tumor cells through the direct interaction of paxillin with LC3. Cell Rep. 2016;15(8):1660-72.

119. Karsli-Uzunbas $\mathrm{G}$, et al. Autophagy is required for glucose homeostasis and lung tumor maintenance. Cancer Discov. 2014;4(8):914-27.

120. Catalano M, et al. Autophagy induction impairs migration and invasion by reversing EMT in glioblastoma cells. Mol Oncol. 2015;9(8):1612-25.

121. La Belle Flynn A, et al. Autophagy inhibition elicits emergence from metastatic dormancy by inducing and stabilizing Pfkfb3 expression. Nat Commun. 2019;10(1):3668.

122. Pietrocola F, et al. Caloric restriction mimetics enhance anticancer immunosurveillance. Cancer Cell. 2016:30(1):147-60.

123. Kimura T, et al. Cellular and molecular mechanism for secretory autophagy. Autophagy. 2017;13(6):1084-5.

124. Lock R, et al. Autophagy-dependent production of secreted factors facilitates oncogenic RAS-driven invasion. Cancer Discov. 2014;4(4):466-79.

125. Hong Z, et al. Ptdlns3P controls mTORC1 signaling through lysosomal positioning. J Cell Biol. 2017;216(12):4217-33.

126. Marat AL, Haucke V. Phosphatidylinositol 3-phosphates-at the interface between cell signalling and membrane traffic. EMBO J. 2016;35(6):561-79.

127. Poteryaev $D$, et al. Identification of the Switch in early-to-late endosome transition. Cell. 2010;141(3):497-508.

128. Bache KG, et al. Hrs regulates multivesicular body formation via ESCRT recruitment to endosomes. J Cell Biol. 2003;162(3):435-42.

129. Futter $C E$, et al. Human VPS34 is required for internal vesicle formation within multivesicular endosomes. J Cell Biol. 2001;155(7):1251-64.

130. Xu Y, et al. SNX3 regulates endosomal function through its PX-domainmediated interaction with Ptdlns(3)P. Nat Cell Biol. 2001;3(7):658-66.

131. Knævelsrud $\mathrm{H}$, et al. Membrane remodeling by the PX-BAR protein SNX18 promotes autophagosome formation. J Cell Biol. 2013;202(2):331-49.

132. Bechtel $W$, et al. Vps34 deficiency reveals the importance of endocytosis for podocyte homeostasis. J Am Soc Nephrol. 2013;24(5):727-43.

133. Siddhanta U, et al. Distinct roles for the p110alpha and hVPS34 phosphatidylinositol 3'-kinases in vesicular trafficking, regulation of the actin cytoskeleton, and mitogenesis. J Cell Biol. 1998;143(6):1647-59.

134. Nascimbeni AC, Codogno P, Morel E. Phosphatidylinositol-3-phosphate in the regulation of autophagy membrane dynamics. FEBS J. 2017:284(9):1267-78.

135. Ketel $\mathrm{K}$, et al. A phosphoinositide conversion mechanism for exit from endosomes. Nature. 2016;529(7586):408-12.

136. Miranda AM, Di Paolo G. Endolysosomal dysfunction and exosome secretion: implications for neurodegenerative disorders. Cell Stress. 2018;2(5):115-8

137. Law F, et al. The VPS34 PI3K negatively regulates RAB-5 during endosome maturation. J Cell Sci. 2017;130(12):2007-17.

138. Jaber N, et al. Vps34 regulates Rab7 and late endocytic trafficking through recruitment of the GTPase-activating protein Armus. J Cell Sci. 2016;129(23):4424-35
139. Baietti MF, et al. Syndecan-syntenin-ALIX regulates the biogenesis of exosomes. Nat Cell Biol. 2012;14(7):677-85.

140. Sapmaz A, et al. USP32 regulates late endosomal transport and recycling through deubiquitylation of Rab7. Nat Commun. 2019;10(1):1454.

141. Odorizzi G, Babst M, Emr SD. Fab1 p Ptdlns(3)P 5-kinase function essential for protein sorting in the multivesicular body. Cell. 1998:95(6):847-58.

142. Falguières T, et al. In vitro budding of intralumenal vesicles into late endosomes is regulated by Alix and Tsg101. Mol Biol Cell. 2008;19(11):4942-55.

143. Hikita T, et al. Src in endosomal membranes promotes exosome secretion and tumor progression. Sci Rep. 2019;9(1):3265.

144. Ghossoub R, et al. Syntenin-ALIX exosome biogenesis and budding into multivesicular bodies are controlled by ARF6 and PLD2. Nat Commun. 2014;5:3477.

145. Ostrowski M, et al. Rab27a and Rab27b control different steps of the exosome secretion pathway. Nat Cell Biol. 2010;12(1):19-30 sup pp 1-13.

146. Hoshino $D$, et al. Exosome secretion is enhanced by invadopodia and drives invasive behavior. Cell Rep. 2013:5(5):1159-68.

147. Yang L, et al. Long non-coding RNA HOTAIR promotes exosome secretion by regulating RAB35 and SNAP23 in hepatocellular carcinoma. Mol Cancer. 2019;18(1):78

148. Yang $L$, et al. The long noncoding RNA HOTAIR activates autophagy by upregulating ATG3 and ATG7 in hepatocellular carcinoma. Mol BioSyst. 2016;12(8):2605-12.

149. Peng $X$, et al. LINC00511 drives invasive behavior in hepatocellular carcinoma by regulating exosome secretion and invadopodia formation. J Exp Clin Cancer Res. 2021;40(1):183.

150. Agbana YL, et al. LINC00511 as a prognostic biomarker for human cancers: a systematic review and meta-analysis. BMC Cancer. 2020;20(1):682.

151. Dong $X$, et al. Long noncoding RNA LINC00511 regulates the proliferation, apoptosis, invasion and autophagy of trophoblast cells to mediate pre-eclampsia progression through modulating the miR-31-5p/homeobox protein A7 axis. J Obstet Gynaecol Res. 2020;46(8):1298-309.

152. Sinha $\mathrm{S}$, et al. Cortactin promotes exosome secretion by controlling branched actin dynamics. J Cell Biol. 2016;214(2):197-213.

153. Villarroya-Beltri C, et al. ISGylation controls exosome secretion by promoting lysosomal degradation of MVB proteins. Nat Commun. 2016;7:13588

154. Majumder P, Chakrabarti O. Mahogunin regulates fusion between amphisomes/MVBs and lysosomes via ubiquitination of TSG101. Cell Death Dis. 2015;6(11):e1970.

155. Buschow SI, et al. MHC II in dendritic cells is targeted to lysosomes or T cell-induced exosomes via distinct multivesicular body pathways. Traffic. 2009;10(10):1528-42.

156. Möbius W, et al. Immunoelectron microscopic localization of cholesterol using biotinylated and non-cytolytic perfringolysin O. J Histochem Cytochem. 2002;50(1):43-55.

157. Möbius W, et al. Recycling compartments and the internal vesicles of multivesicular bodies harbor most of the cholesterol found in the endocytic pathway. Traffic. 2003:4:222-31.

158. Jordens I, et al. The Rab7 effector protein RILP controls lysosomal transport by inducing the recruitment of dynein-dynactin motors. Curr Biol. 2001;11(21):1680-5.

159. Jongsma ML, et al. SKIP-HOPS recruits TBC1D15 for a Rab7-toArl8b identity switch to control late endosome transport. EMBO J. 2020;39(6):e102301.

160. Guo H, et al. Atg5 disassociates the V1V0-ATPase to promote exosome production and tumor metastasis independent of canonical macroautophagy. Dev Cell. 2017:43(6):716-730.e7.

161. Savina A, et al. Rab11 promotes docking and fusion of multivesicular bodies in a calcium-dependent manner. Traffic. 2005;6(2):131-43.

162. Wang $\mathrm{K}$, et al. Mechanical stress-dependent autophagy component release via extracellular nanovesicles in tumor cells. ACS Nano. 2019;13(4):4589-602.

163. Fader CM, et al. TI-VAMPNAMP7 and VAMP3/cellubrevin: two v-SNARE proteins involved in specific steps of the autophagy/multivesicular body pathways. Biochim Biophys Acta. 2009:1793(12):1901-16. 
164. Lefebvre C, Legouis R, Culetto E. ESCRT and autophagies: Endosomal functions and beyond. Semin Cell Dev Biol. 2018;74:21-8.

165. Chen Y-D, et al. Exophagy of annexin A2 via RAB11, RAB8A and RAB27A in IFN- $\gamma$-stimulated lung epithelial cells. Sci Rep. 2017;7(1):5676.

166. Bader $\mathrm{CA}$, et al. Atg9 is required for intraluminal vesicles in amphisomes and autolysosomes. Biol Open. 2015;4(11):1345-55.

167. Moreau K, et al. Autophagosome precursor maturation requires homotypic fusion. Cell. 2011;146(2):303-17.

168. Fader CM, Aguilera MO, Colombo MI. ATP is released from autophagic vesicles to the extracellular space in a VAMP7-dependent manner. Autophagy. 2012;8(12):1741-56.

169. Jeppesen DK, et al. Reassessment of exosome composition. Cell. 2019;177(2):428-445.e18.

170. Baixauli F, López-Otín C, Mittelbrunn M. Exosomes and autophagy: coordinated mechanisms for the maintenance of cellular fitness. Front Immunol. 2014:5:403.

171. Omi J, et al. The inducible amphisome isolates viral hemagglutinin and defends against influenza A virus infection. Nat Commun 2020;11(1):162.

172. Kraya AA, et al. Identification of secreted proteins that reflect autophagy dynamics within tumor cells. Autophagy. 2015;11(1):60-74.

173. Dupont N, et al. Autophagy-based unconventional secretory pathway for extracellular delivery of IL-1 $\beta$. EMBO J. 2011;30(23):4701-11.

174. Zhang $M$, et al. Translocation of interleukin-1 $\beta$ into a vesicle intermediate in autophagy-mediated secretion. Elife. 2015;4.

175. New J, Thomas SM. Autophagy-dependent secretion: mechanism, factors secreted, and disease implications. Autophagy. 2019;15(10):1682-93.

176. Kimura T, et al. Dedicated SNAREs and specialized TRIM cargo receptors mediate secretory autophagy. EMBO J. 2017;36(1):42-60.

177. Qiang L, et al. Autophagy gene ATG7 regulates ultraviolet radiation-induced inflammation and skin tumorigenesis. Autophagy. 2017:13(12):2086-103

178. Peng $X$, et al. IKK $\beta$ activation promotes amphisome formation and extracellular vesicle secretion in tumor cells. Biochim Biophys Acta Mol Cell Res. 1868;2021(1):1 18857.

179. Maycotte P, et al. Autophagy supports breast cancer stem cell maintenance by regulating IL6 secretion. Mol Cancer Res. 2015;13(4):651-8.

180. Huang S-C, et al. Discovery and optimization of pyrazolopyrimidine sulfamates as ATG7 inhibitors. Bioorg Med Chem. 2020;28(19):115681.

181. King HW, Michael MZ, Gleadle JM. Hypoxic enhancement of exosome release by breast cancer cells. BMC Cancer. 2012:12:421

182. Daskalaki I, Gkikas I, Tavernarakis N. Hypoxia and selective autophagy in cancer development and therapy. Front Cell Dev Biol. 2018;6(104).

183. Kanemoto $S$, et al. Multivesicular body formation enhancement and exosome release during endoplasmic reticulum stress. Biochem Biophys Res Commun. 2016;480(2):166-72.

184. Bhattacharya S, et al. GAIP interacting protein C-terminus regulates autophagy and exosome biogenesis of pancreatic cancer through metabolic pathways. PLoS One. 2014;9(12):e114409.

185. Muders MH, et al. Targeting GIPC/synectin in pancreatic cancer inhibits tumor growth. Clin Cancer Res. 2009;15(12):4095-103.

186. Dutta S, et al. Interactions between exosomes from breast cancer cells and primary mammary epithelial cells leads to generation of reactive oxygen species which induce DNA damage response, stabilization of p53 and autophagy in epithelial cells. PLoS One. 2014;9(5):e97580.

187. Wu Q, et al. Exosomes from the tumour-adipocyte interplay stimulate beige/brown differentiation and reprogram metabolism in stromal adipocytes to promote tumour progression. J Exp Clin Cancer Res. 2019:38(1):223.

188. Xu J, et al. Hypoxic glioma-derived exosomes promote M2-like macrophage polarization by enhancing autophagy induction. Cell Death Dis. 2021;12(4):373.

189. Yang Y, et al. Acquisition of new tumor cell properties by MSC-derived exosomes. Int J Oncol. 2015;47(1):244-52.

190. Kucharewicz K, et al. Simultaneous induction and blockade of autophagy by a single agent. Cell Death Dis. 2018;9(3):353.

191. Chen Y, et al. Increased interleukin-6 levels in the astrocyte-derived exosomes of sporadic amyotrophic lateral sclerosis patients. Front Neurosci. 2019;13(574).
192. Im K, et al. The comparison of exosome and exosomal cytokines between young and old individuals with or without gastric cancer. Int J Gerontol. 2018:12(3):233-8.

193. Qin B, et al. IL-6 Inhibits Starvation-induced Autophagy via the STAT3/ Bcl-2 Signaling Pathway. Sci Rep. 2015;5:15701.

194. Linnemann A, et al. Interleukin 6 protects pancreatic $\beta$ cells from apoptosis by stimulation of autophagy. FASEB J. 2017:31.

195. Frühbeis $C$, et al. Physical exercise induces rapid release of small extracellular vesicles into the circulation. J Extracell Vesicles. 2015:4(1):28239.

196. Follo C, et al. Inhibition of autophagy initiation potentiates chemosensitivity in mesothelioma. Mol Carcinog. 2018;57(3):319-32.

197. Zachari M, Ganley IG. The mammalian ULK1 complex and autophagy initiation. Essays Biochem. 2017;61(6):585-96.

198. Petherick KJ, et al. Pharmacological inhibition of ULK1 kinase blocks mammalian target of rapamycin (mTOR)-dependent autophagy. J Biol Chem. 2015;290(18):11376-83.

199. Young ARJ, et al. Starvation and ULK1-dependent cycling of mammalian Atg9 between the TGN and endosomes. J Cell Sci. 2006;119(18):3888-900

200. Urano Y, et al. 6-Hydroxydopamine induces secretion of PARK7/DJ-1 via autophagy-based unconventional secretory pathway. Autophagy. 2018;14(11):1943-58.

201. Scotto Rosato A, et al. TRPML1 links lysosomal calcium to autophagosome biogenesis through the activation of the CaMKK $\beta / N P S 34$ pathway. Nat Commun. 2019;10(1):5630

202. Zhou X, et al. Unc-51-like kinase 1/2-mediated endocytic processes regulate filopodia extension and branching of sensory axons. Proc Natl Acad Sci. 2007;104(14):5842-7.

203. Chen Y, et al. Dual targeting of NUAK1 and ULK1 using the multitargeted inhibitor MRT68921 exerts potent antitumor activities. Cell Death Dis. 2020;11(8):712.

204. Palamiuc L, Ravi A, Emerling BM. Phosphoinositides in autophagy: current roles and future insights. FEBS J. 2020;287(2):222-38.

205. Zhou C, et al. The heme oxygenase-1 inhibitor ZnPPIX induces non-canonical, Beclin 1-independent, autophagy through p38 MAPK pathway. Acta Biochim Biophys Sin. 2012;44:815-22.

206. Wu YT, et al. Dual role of 3-methyladenine in modulation of autophagy via different temporal patterns of inhibition on class I and III phosphoinositide 3-kinase. J Biol Chem. 2010;285(14):10850-61.

207. Xing C, et al. Class I phosphatidylinositol 3-kinase inhibitor LY294002 activates autophagy and induces apoptosis through p53 pathway in gastric cancer cell line SGC7901. Acta Biochim Biophys Sin Shanghai. 2008;40(3):194-201

208. Seglen PO, Gordon PB. 3-Methyladenine: specific inhibitor of autophagic/lysosomal protein degradation in isolated rat hepatocytes. Proc Natl Acad Sci U S A. 1982;79(6):1889-92.

209. Lelouard $\mathrm{H}$, et al. Regulation of translation is required for dendritic cell function and survival during activation. J Cell Biol. 2007;179(7):1427-39.

210. Chicote $\mathrm{J}$, et al. Cell death triggered by the autophagy inhibitory drug 3 -methyladenine in growing conditions proceeds with DNA damage. Front Pharmacol. 2020;11:580343. https://doi.org/10.3389/fphar.2020. 580343

211. Caro LH, et al. 3-Methyladenine, an inhibitor of autophagy, has multiple effects on metabolism. Eur J Biochem. 1988;175(2):325-9.

212. Korolchuk VI, et al. Autophagy inhibition compromises degradation of ubiquitin-proteasome pathway substrates. Mol Cell. 2009;33(4):517-27.

213. Zhang H-G, Grizzle WE. Exosomes: a novel pathway of local and distant intercellular communication that facilitates the growth and metastasis of neoplastic lesions. Am J Pathol. 2014;184(1):28-41.

214. Zhang J, et al. Exosomes/tricalcium phosphate combination scaffolds can enhance bone regeneration by activating the PI3K/Akt signaling pathway. Stem Cell Res Ther. 2016;7:136.

215. Clayton A, et al. Analysis of antigen presenting cell derived exosomes, based on immuno-magnetic isolation and flow cytometry. J Immunol Methods. 2001:247(1-2):163-74.

216. Llorente A, de Marco MAC, Alonso MA. Caveolin-1 and MAL are located on prostasomes secreted by the prostate cancer PC-3 cell line. J Cell Sci. 2004:117(22):5343-51. 
217. Abdulrahman BA, Abdelaziz DH, Schatzl HM. Autophagy regulates exosomal release of prions in neuronal cells. J Biol Chem. 2018;293(23):8956-68

218. Bright NA, et al. The relationship between lumenal and limiting membranes in swollen late endocytic compartments formed after wortmannin treatment or sucrose accumulation. Traffic. 2001;2(9):631-42.

219. Isosaki M. Inhibition of wortmannin activities by amino compounds. Biochem Biophys Res Commun. 2004;324(4):1406-12.

220. Iula $L$, et al. Autophagy mediates interleukin-1 $\beta$ secretion in human neutrophils. Front Immunol. 2018;9:269.

221. Harris J, et al. Autophagy controls IL-1 beta secretion by targeting pro-IL1 beta for degradation. J Biol Chem. 2011;286(11):9587-97.

222. Fukao T, et al. PI3K-mediated negative feedback regulation of IL-12 production in DCs. Nat Immunol. 2002;3(9):875-81.

223. Miranda AM, et al. Neuronal lysosomal dysfunction releases exosomes harboring APP C-terminal fragments and unique lipid signatures. Nat Commun. 2018;9(1):291

224. Liu J, et al. Beclin1 controls the levels of p53 by regulating the deubiquitination activity of USP10 and USP13. Cell. 2011;147(1):223-34.

225. Yu X, Harris SL, Levine AJ. The regulation of exosome secretion: a novel function of the p53 protein. Cancer Res. 2006;66(9):4795-801.

226. Liu J, et al. Distinct dasatinib-induced mechanisms of apoptotic response and exosome release in imatinib-resistant human chronic myeloid leukemia cells. Int J Mol Sci. 2016;17(4):531.

227. Wang C, et al. Autophagic lipid metabolism sustains mTORC1 activity in TSC-deficient neural stem cells. Nat Metab. 2019;1(11):1127-40.

228. McKnight NC, et al. Beclin 1 is required for neuron viability and regulates endosome pathways via the UVRAG-VPS34 complex. PLoS Genet. 2014;10(10):e1004626.

229. McKnight NC, Yue Z. Beclin 1, an essential component and master regulator of PI3K-III in health and disease. Curr Pathobiol Rep. 2013;1(4):231-8.

230. Gladue DP, et al. Foot-and-mouth disease virus nonstructural protein $2 \mathrm{C}$ interacts with Beclin1, modulating virus replication. J Virol. 2012;86(22):12080-90.

231. Robke $L$, et al. Phenotypic identification of a novel autophagy inhibitor chemotype targeting lipid kinase VPS34. Angew Chem Int Ed. 2017:56(28):8153-7.

232. Marsh T, Debnath J. Ironing out VPS34 inhibition. Nat Cell Biol. 2014;17:1-3.

233. Ronan B, et al. A highly potent and selective Vps34 inhibitor alters vesicle trafficking and autophagy. Nat Chem Biol. 2014;10(12):1013-9.

234. Wu Y, et al. Synthesis and screening of 3-MA derivatives for autophagy inhibitors. Autophagy. 2013;9(4):595-603.

235. Vats S, Manjithaya R. A reversible autophagy inhibitor blocks autophagosome-lysosome fusion by preventing Stx 17 loading onto autophagosomes. Mol Biol Cell. 2019;30(17):2283-95.

236. Keller MD, et al. Decoy exosomes provide protection against bacterial toxins. Nature. 2020;579(7798):260-4.

237. Cheng X-T, et al. Axonal autophagosomes recruit dynein for retrograde transport through fusion with late endosomes. J Cell Biol. 2015;209(3):377-86

238. Al-Bari A. Chloroquine analogues in drug discovery: New directions of uses, mechanisms of actions and toxic manifestations from malaria to multifarious diseases. J Antimicrob Chemother. 2015;70.

239. Mauthe $M$, et al. Chloroquine inhibits autophagic flux by decreasing autophagosome-lysosome fusion. Autophagy. 2018;14(8):1435-55.

240. Lu S, et al. Lysosomal adaptation: how cells respond to lysosomotropic compounds. PLoS One. 2017;12(3):e0173771

241. Gallagher LE, et al. Lysosomotropism depends on glucose: a chloroquine resistance mechanism. Cell Death Dis. 2017;8(8):e3014.

242. Davis MJ, Swanson JA. Technical advance: Caspase-1 activation and $\mathrm{IL}-1 \beta$ release correlate with the degree of lysosome damage, as illustrated by a novel imaging method to quantify phagolysosome damage. J Leukoc Biol. 2010;88(4):813-22.

243. Orlinska U, Newton RC. Role of glucose in interleukin-1 beta production by lipopolysaccharide-activated human monocytes. J Cell Physiol. 1993;157(1):201-8.

244. Cotzomi-Ortega l, et al. Autophagy inhibition induces the secretion of macrophage migration inhibitory factor (MIF) with autocrine and paracrine effects on the promotion of malignancy in breast cancer. Biology. 2020;9(1):20.

245. Li M, et al. Suppression of lysosome function induces autophagy via a feedback down-regulation of MTOR complex 1 (MTORC1) activity. J Biol Chem. 2013;288(50):35769-80.

246. Deng J, et al. The role of autophagy and amphisomes in virus recognition, TLR 9 recruitment and virus-stimulated IFN-a production by human plasmacytoid dendritic cells (pDC) (45.19). J Immunol. 2012;188(1 Supplement):45.19.

247. Mauvezin C, et al. Autophagosome-lysosome fusion is independent of V-ATPase-mediated acidification. Nat Commun. 2015:6:7007.

248. van Deurs B, Holm PK, Sandvig K. Inhibition of the vacuolar H(+)ATPase with bafilomycin reduces delivery of internalized molecules from mature multivesicular endosomes to lysosomes in HEp-2 cells. Eur J Cell Biol. 1996;69(4):343-50.

249. van Weert AW, et al. Transport from late endosomes to lysosomes, but not sorting of integral membrane proteins in endosomes, depends on the vacuolar proton pump. J Cell Biol. 1995;130(4):821-34.

250. Zou W, et al. Exosome release is regulated by mTORC1. Adv Sci. 2019;6(3):1801313.

251. Palokangas $\mathrm{H}$, et al. Retrograde transport from the pre-Golgi intermediate compartment and the Golgi complex is affected by the vacuolar H+-ATPase inhibitor bafilomycin A1. Mol Biol Cell. 1998;9(12):3561-78.

252. Chen Q, et al. Knockdown of Parkinson's disease-related gene ATP13A2 reduces tumorigenesis via blocking autophagic flux in colon cancer. Cell Biosci. 2020;10(1):144

253. Bento CF, et al. The Parkinson's disease-associated genes ATP13A2 and SYT11 regulate autophagy via a common pathway. Nat Commun. 2016;7(1):11803.

254. Kong SM, et al. Parkinson's disease-linked human PARK9/ATP13A2 maintains zinc homeostasis and promotes a-Synuclein externalization via exosomes. Hum Mol Genet. 2014;23(11):2816-33.

255. Minakaki G, et al. Autophagy inhibition promotes SNCA/alphasynuclein release and transfer via extracellular vesicles with a hybrid autophagosome-exosome-like phenotype. Autophagy. 2018;14(1):98-119.

256. Sharma G, et al. A family of PIKFYVE inhibitors with therapeutic potential against autophagy-dependent cancer cells disrupt multiple events in lysosome homeostasis. Autophagy. 2019;15(10):1694-718.

257. Dong XP, et al. PI (3,5)P(2) controls membrane trafficking by direct activation of mucolipin $\mathrm{Ca}(2+)$ release channels in the endolysosome. Nat Commun. 2010;1(4):38.

258. Li SC, et al. The signaling lipid $\mathrm{PI}(3,5) \mathrm{P}_{2}$ stabilizes $\mathrm{V}_{1}-\mathrm{V}(\mathrm{0})$ sector interactions and activates the V-ATPase. Mol Biol Cell. 2014;25(8):1251-62.

259. Hessvik NP, et al. PIKfyve inhibition increases exosome release and induces secretory autophagy. Cell Mol Life Sci. 2016;73(24):4717-37.

260. Ji X, Zhang X, Li Z. ULK1 inhibitor induces spindle microtubule disorganization and inhibits phosphorylation of Ser 10 of histone H3. FEBS Open Biol. 2020;10(11):2452-63.

261. Karve $S$, et al. Revival of the abandoned therapeutic wortmannin by nanoparticle drug delivery. Proc Natl Acad Sci U S A. 2012;109(21):8230-5.

262. Guo J, et al. Potent USP10/13 antagonist spautin-1 suppresses melanoma growth via ROS-mediated DNA damage and exhibits synergy with cisplatin. J Cell Mol Med. 2020;24(7):4324-40.

263. Pellegrini $\mathrm{P}$, et al. Acidic extracellular pH neutralizes the autophagyinhibiting activity of chloroquine: implications for cancer therapies. Autophagy. 2014;10(4):562-71.

264. Collins KP, Jackson KM, Gustafson DL. Hydroxychloroquine: A Physiologically-Based Pharmacokinetic Model in the Context of Cancer-Related Autophagy Modulation. J Pharmacol Exp Ther. 2018;365(3):447-59.

265. Bayer $\mathrm{N}$, et al. Effect of bafilomycin $\mathrm{A} 1$ and nocodazole on endocytic transport in HeLa cells: implications for viral uncoating and infection. J Virol. 1998;72(12):9645-55.

\section{Publisher's Note}

Springer Nature remains neutral with regard to jurisdictional claims in published maps and institutional affiliations. 